\title{
Absence of Diffusion in the Anderson Tight Binding Model for Large Disorder or Low Energy
}

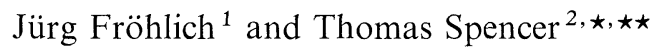 \\ 1 Theoretical Physics, ETH. Hönggerberg, CH-8093 Zürich, Switzerland \\ 2 Department of Mathematics, California Institute of Technology, Pasadena, CA 91125, USA
}

\begin{abstract}
We prove that the Green's function of the Anderson tight binding Hamiltonian decays exponentially fast at long distances on $\mathbb{Z}^{v}$, with probability 1 . We must assume that either the disorder is large or the energy is sufficiently low. Our proof is based on perturbation theory about an infinite sequence of block Hamiltonians and is related to KAM methods.
\end{abstract}

\section{Introduction}

\subsection{General Background}

In this paper we analyze the Schrödinger operator (Hamiltonian)

$$
H=H(v)=-\Delta+v,
$$

where $\Delta$ is the finite difference Laplacian on $\mathbb{Z}^{v}$, and $v=\{v(j)\}$ is a random potential. We shall consider the case in which the $v(j)$ are independent (e.g. Gaussian) random variables with mean 0 and variance $\gamma$. The Hamiltonian (1.1) was introduced by Anderson [1] to model the dynamics of a quantum mechanical particle - the electron - moving in a random medium. The random medium may be thought of as a crystal with impurities of random strength $v(j)$. The variance $\gamma$ measures the overall strength of the impurities or of the disorder. In this model the electron interacts only with the impurities. Electron-electron interactions and thermal effects are neglected.

Let $\psi_{t}=e^{i t H} \psi_{0}$ be the time evolution of a wave function $\psi_{0}$ supported near the origin, e.g. $\psi_{0}(j)=$ const $e^{-|j|}$. In order to describe the long time behaviour of our particle, consider the spread of $\psi_{t}$ as measured by

$$
r^{2}(t) \equiv \sum_{x \in \mathbb{Z}^{v}} x^{2}\left|\psi_{t}(x)\right|^{2} .
$$

* Permanent address: Courant Institute, 251 Mercer, New York, NY 10012, USA

$\star \star$ Work supported in part by NSF Grant DMR 8100417 and by Grant PHY 82-03669 
The quantity $r^{2}(t)$ represents the mean square of the distance of the particle from the origin at time $t$. In three or more dimensions and for small disorder $\gamma$, the particle is expected to diffuse with probability 1 . In other words, except for a set of potentials $v$ of measure 0 ,

$$
r^{2}(t) \cong D t, D>0 \text {. }
$$

Here $D$ represents the diffusion constant which depends on $v$ and $\psi_{0}$. In solid state theory, $D$ is proportional to the conductivity. It is easy to show that if we set $v \equiv 0$ or equal to a fixed periodic potential, $r^{2}(t) \approx$ const $t^{2}$, hence there is infinite conductivity for such a system. (A proof of this behaviour can be based on analyzing the Heisenberg equations of motion.)

The main purpose of this paper is to prove that if the disorder $\gamma$ is sufficiently large, then the diffusion constant $D$ and the conductivity vanish with probability 1 . If the disorder is small we still prove the absence of diffusion for sufficiently low energy $E$. This means that if the wave function $\psi_{0}$ in (1.2) belongs to the spectral subspace of states characterized by $|H-E| \leqq \kappa$, with $|E|$ large enough and $\kappa \leqq 1$, then $D \equiv D(E)=0$. See (1.15)-(1.18) for the precise definition of $D$ and of $D(E)$ used in our paper.

Anderson [1] argued that, for large disorder, the particle is trapped or localized near the origin, i.e.

$$
r^{2}(t) \leqq \text { const, uniformly in } t .
$$

While our results do not imply localization, we hope that some variant of the methods described in this paper will yield this result.

In one dimension, localization has been established for all strictly positive values of $\gamma$ (see [2-5]). Moreover it is known that $H$ has dense pure point spectrum with eigenfunctions which decay exponentially about some point in space. The simplest example of this phenomenon is given by the degenerate case $H=v$ : Clearly $r^{2}(t) \leqq$ const, and the eigenfunctions are simply (Kronecker) delta functions.

In three dimensions and for weak disorder, the spectrum of $H$ is expected to consist of a band of absolutely continuous spectrum filling out an interval $\left(m, m^{\prime}\right)$, outside which the spectrum is dense pure point. The numbers $m, m^{\prime}$ are called mobility edges. Recently, Kunz and Souillard [6] have established the existence of absolutely continuous spectrum when $\mathbb{Z}^{v}$ is replaced by the Cayley tree. They have also proven results on the typical decay rate of eigenfunctions for energies near the mobility edges.

The main estimates of this paper concern the decay of the Green's function

$$
G(E+i \varepsilon, v ; x, y)=[H-E-i \varepsilon]^{-1}(x, y),
$$

for large $\gamma$, or for $|E-2 v| \gg 1$. Roughly speaking, we establish exponential decay of $G$ in $|x-y|$, provided $v$ is excluded from a set of measure $\alpha|x-y|^{-p}$. The constant $p$ can be made arbitrarily large by increasing $|E-2 v|$ or $\gamma$. The diffusion constant $D(E)$ and conductivity have expressions in terms of the Green's function, known as the Kubo formulae. The methods we use to derive estimates on $G$ are related to those of the KAM scheme for handling small divisors. In fact, small divisors appear in the resolvent because eigenvalues of $H$ come arbitrarily close to $E$. We also rely heavily on techniques we have developed to prove phase transitions in the 
$1 / r^{2}$ Ising model [7] and in the two-dimensional Coulomb gas [8]. However, no knowledge of these papers is assumed.

Our proof is based on perturbation theory about an infinite sequence of "block" Hamiltonians. The "blocks" correspond to regions of $\mathbb{Z}^{v}$ on which the potential is singular, in the sense that the "block" Hamiltonian has eigenvalues close to $E$. In order to decouple a "block" of singular sites, Dirichlet boundary conditions are imposed, thereby decoupling $H$ along the boundary of the region. The regions are chosen depending on the particular configuration $v$. We inductively use information obtained for small blocks to obtain results for larger blocks. As the block size increases, the eigenvalues, $E_{i}$, of the block Hamiltonian are permitted to get closer to $E$. The divergence arising from small divisors $\left(E-E_{i}\right)^{-1}$ will be offset by small factors arising from the Green's function over long distances proportional to the length of the block.

Finally we remark that our analysis applies not only to the Gaussian but to a wide class of distributions. What is important for our analysis is that the density of states $\varrho(E)$ defined by $(1.11)$ be bounded and small.

\subsection{Definitions and Notation}

The state space for a quantum mechanical particle moving in $\mathbb{Z}^{v}$ is given by $l_{2}\left(\mathbb{Z}^{v}\right)$ the space of square summable functions $f(j), j \in \mathbb{Z}^{v}$. The kinetic energy operator of such a particle is the finite difference Laplacian which is the bounded operator on $l_{2}\left(\mathbb{Z}^{v}\right)$ defined by

$$
(\Delta f)(j) \equiv \sum_{i:|i-j|=1}(f(i)-f(j)) .
$$

Thus, the Hamiltonian $H(v)$, given by (1.1), is a self-adjoint operator defined on the domain of the potential $v$. Hence the Green's function (the matrix elements of the resolvent) of $H$, given by (1.4), is well defined for $\varepsilon \neq 0$.

Let spec $H \equiv \sigma(H)$ denote the spectrum of $H, \sigma_{p p}(H)$ the pure point spectrum, and $\sigma_{c}(H)$ the continuous spectrum of $H$.

The random potential $v$ belongs to the probability space

$$
\Omega=\prod_{j \in \mathbb{Z}^{v}}(\mathbb{R}, d \lambda(v(j))),
$$

where $d \lambda(v)$ is a probability measure on the reals with a bounded density with respect to Lebesgue measure. We set

$$
d P(v) \equiv \prod_{j \in \mathbb{Z}^{v}} d \lambda(v(j))
$$

Note that the group of translations acts ergodically on $\Omega$ and leaves the spectrum of $H$ invariant. Consequently it can be shown, see [3], that with probability one

$$
\sigma(H(v))=[0,4 v]+\operatorname{supp} d \lambda,
$$

and that $\sigma_{p p}(H(v))$ and $\sigma_{c}(H(v))$ are independent of $v, d P$-almost everywhere.

Let $\Lambda$ be some subset of $\mathbb{Z}^{v}$. We define $\Delta_{\Lambda}$ to be the finite difference Laplacian with zero Dirichlet data on the boundary $\partial \Lambda$ ( $\equiv$ the set of links connecting sites in 
$\Lambda$ to sites in the complement, $\sim \Lambda$, of $\Lambda$ ). Let

$$
H_{\Lambda}(v) \equiv-\Delta_{\Lambda}+v \chi_{\Lambda},
$$

where $\chi_{\Lambda}$ is the characteristic function of $\Lambda$. This operator acts on the Hilbert space $l_{2}(\Lambda)$. If $\Lambda$ is finite $l_{2}(\Lambda) \cong \mathbb{C}^{|\Lambda|}$, where $|\Lambda|$ is the number of sites in $\Lambda$, is finite dimensional, and the spectrum of $H_{A}(v)$ is discrete. This permits us to define $N_{\Lambda}(E, v)$ as the number of eigenvalues of $H_{\Lambda}(v)$ less than, or equal to $E$.

By a simple argument involving the ergodic theorem (see e.g. [9])

$$
\lim _{\Lambda \uparrow \mathbb{Z}^{v}} \frac{1}{|\Lambda|} N_{\Lambda}(E, v) \equiv N(E)
$$

exists and is independent of $v$, almost surely. Under our assumptions, the density of states

$$
\varrho(E) \equiv \frac{d N(E)}{d E}
$$

exists as a bounded function. In fact, Wegner [10] has shown that (see Appendix C)

$$
\varrho(E) \leqq \sup _{v} \frac{d \lambda(v)}{d v}
$$

and

$$
\varrho(E)>0, \text { for } E \in \sigma(H) \text {. }
$$

If $\frac{d \lambda}{d v}$ has certain analyticity properties, e.g.

$$
\frac{d \lambda(v)}{d v}=(2 \pi \gamma)^{-1 / 2} \exp \left[-v^{2} / 2 \gamma\right]
$$

then $\varrho(E)$ is analytic in $E$ when either $\gamma \gg 1$, or $|E-2 v| \gg 1$ (see [9]).

Next, we turn to the formal definition of the diffusion constant and the conductivity, as given by Kubo (see e.g. [11]):

$$
\begin{gathered}
\varrho(E) D(E) \equiv \lim _{\varepsilon \downarrow 0} \frac{2 \varepsilon^{2}}{\pi v} \sum_{x}|x|^{2} \cdot \int d P(v)|G(E+i \varepsilon, v ; 0, x)|^{2}, \\
D \equiv \int \varrho(E) D(E) d E,
\end{gathered}
$$

and the conductivity is given by

$$
\sigma(E)=e^{2} \varrho(E) D(E),
$$

where $e$ is the electric charge.

To relate the time-independent definition of $D(E), D$, in terms of the Green's function of $H$, to the time-dependent definitions (1.3), we choose

$$
\psi_{0}(j)=\delta(j, 0) \text {, }
$$

and note that if $f(t) / t \rightarrow D$, as $t \rightarrow \infty$, for some function $f(t)$ defined on $[0, \infty)$, then

$$
D=\lim _{\varepsilon \downarrow 0} \varepsilon^{2} \int_{0}^{\infty} e^{-\varepsilon t} f(t) d t,
$$


and formally,

$$
\varepsilon^{2} \int_{0}^{\infty} e^{-\varepsilon t}\left|\left(e^{-i t H} \psi_{0}\right)(x)\right|^{2} d t=\frac{\varepsilon^{2}}{2 \pi} \int d E\left|G\left(E+\frac{i \varepsilon}{2}, v ; 0, x\right)\right|^{2} d t .
$$

It is not hard to show that if $D$, as given by (1.15), (1.16), vanishes, an ergodic mean of $r^{2}(t) / t$ vanishes [i.e. existence of the limit of $r^{2}(t) / t$, as $t \rightarrow \infty$, is not required in the above arguments].

Before summarizing our main results we recall, for completeness, a convenient sufficient condition for the existence of point spectrum, essentially due to Ruelle $[12]^{1}$ :

If $f(t)$ is a non-negative function on $[0, \infty)$ and $\varepsilon=T^{-1}$ then

$$
\begin{aligned}
\frac{1}{T} \int_{0}^{T} f(t) d t & \leqq \varepsilon \int_{0}^{\varepsilon-1} e^{1-\varepsilon t} f(t) d t \\
& \leqq e \varepsilon \int_{0}^{\infty} e^{-\varepsilon t} f(t) d t
\end{aligned}
$$

Let $\mathscr{E}_{c}$ denote the projection onto the spectral subspace of $H$ corresponding to $\sigma_{c}(H)$, and let $P_{|x| \geqq R}$ be the projection onto wave functions which vanish in $\left\{x \in \mathbb{Z}^{v}:|x|<R\right\}$. Let $h(R)$ be a positive function, with $h(R) \rightarrow+\infty$, as $R \rightarrow \infty$. Then, using (1.19) and results of $[12,3]$,

$$
\begin{aligned}
\left\|\mathscr{E}_{c} \psi\right\|^{2} & =\lim _{R \rightarrow \infty} \lim _{T \rightarrow \infty} \frac{1}{T} \int_{0}^{T}\left\|P_{|x| \geqq R} e^{i t H} \psi\right\|^{2} d t \\
& \leqq e \lim _{R \rightarrow \infty} \varlimsup_{\varepsilon \downarrow 0} \varepsilon \int_{0}^{\infty} e^{-\varepsilon t}\left\|P_{|x| \geqq R} e^{-i t H} \psi\right\|^{2} d t \\
& \leqq e \varlimsup_{R \rightarrow \infty}(1 / h(R)) \varlimsup_{\varepsilon \downarrow 0} \varepsilon \int_{0}^{\infty} e^{-\varepsilon t}\left\|\sqrt{h(|x|)} e^{-i t H} \psi\right\|^{2} d t .
\end{aligned}
$$

By arguments similar to the ones used to obtain (1.18) we conclude from (1.20) that in order to show that, for $d P$-almost every $v$,

$$
\sigma(H) \cap I=\sigma_{p p}(H), \text { i.e. } \sigma_{c}(H) \cap I=\emptyset,
$$

for some interval $I$, it suffices to show that

$$
\varepsilon \sum_{x} h(|x|) \int_{I} d E \int_{\Omega} d P(v)|G(E+i \varepsilon, v ; 0, x)|^{2}
$$

remains bounded, as $\varepsilon \downarrow 0$. This, however, will not follow from the results proven in this paper, no matter how $I$ is chosen.

\subsection{Main Theorems}

We now summarize the main results of this paper. Let $\delta$ be a measure of the disorder given by

$$
\delta^{-1} \equiv \sup (d \lambda(v) / d v) .
$$

1 We thank B. Simon for explaining to us Ruelle's criterion in the form stated here which is essentially in [3] 
Theorem 1.1. 1) For large disorder $\delta, D(E)=0$, for all $E$.

2) If $d \lambda(v)$ is given by the Gaussian distribution (1.14) $(\delta=\sqrt{2 \pi \gamma})$ then there is a finite constant $E_{\gamma}$ such that $D(E)=0$, for $|E-2 v|>E_{\gamma}$.

Remarks. (1) Theorem 1.1 holds in arbitrary dimension $v$. Our estimates for $E_{\gamma}$ approach $2 v$ as $\gamma \downarrow 0$ since the density of states $\varrho(E)$ goes to 0 for $|E-2 v|>2 v$ as $\gamma \downarrow 0$.

(2) A result analogous to Theorems 1.1 and 1.2 holds for a large class of distributions $d \lambda(v)$ briefly described in Appendix C.

Theorem 1.1 is a simple corollary of the following more general result.

Theorem 1.2. There are constants $\lambda(p, m)$ and $E(\gamma, p, m)$ such that, for every $m<x$ and every $p<x$,

$$
\operatorname{Prob}\left\{v:|G(E+i \varepsilon, v ; 0, x)| \leqq e^{m(N-|x|)}, \varepsilon \neq 0\right\} \geqq 1-C_{p} / N^{p},
$$

uniformly in $\varepsilon \neq 0$, for some finite constant $C_{p}$, provided $\delta^{-1} \leqq \lambda(p, m)$ or $|E-2 v|$ $\geqq E(\gamma, p, m)$ (when di is Gaussian), respectively.

Remark. We have used (and shall use) the convention that

$$
\text { Prob } V \equiv \int_{V} d P(v) \equiv P(V),
$$

where $V$ is some measurable subset of $\Omega$.

Next we show that Theorem 1.2 implies Theorem 1.1. We define

$$
V_{N} \equiv\left\{v:|G(E+i \varepsilon, v ; 0, x)| \leqq e^{m(N-|x|)}, \varepsilon \neq 0\right\} .
$$

By Eq. (1.15)

$$
\varrho(E) D(E)=\lim _{\varepsilon \downarrow 0} \frac{2 \varepsilon^{2}}{\pi v} \sum_{x}|x|^{2} \int|G(E+i \varepsilon, v ; 0, x)|^{2} d P(v) .
$$

We decompose $\mathbb{Z}^{v}$ into annuli, $A_{j}, j=0,1,2, \ldots$, where, for some constant $R$ to be chosen later,

$$
\begin{aligned}
& A_{0} \equiv\{x:|x|<R\}, \\
& A_{j} \equiv\left\{x: R 2^{j-1} \leqq|x|<R 2^{j}\right\}, \quad j=1,2,3, \ldots .
\end{aligned}
$$

Then, using the definition of $V_{N}$, we get

$\varrho(E) D(E) \leqq \lim _{\varepsilon \downarrow 0} \frac{2}{\pi v} \sum_{j=0}^{\prime}\left\{\varepsilon^{2} e^{m N_{J}} P\left(V_{N_{j}}\right)\left[\sum_{x \in A_{j}}|x|^{2} e^{-m|x|}\right]+c_{v}\left(R 2^{j}\right)^{v+2} P\left(\sim V_{N_{J}}\right)\right\}$,

where $c_{v}$ is a constant ( $\approx$ volume of the unit ball in $\left.\mathbb{R}^{v}\right)$, and in the second term on the right side of (1.25) we have used the trivial upper bound

$$
\varepsilon^{2}|G(E+i \varepsilon, v ; 0, x)|^{2} \leqq 1 ;
$$

the sequence $\left(N_{j}\right)_{j=0}^{\infty}$ can be chosen at convenience. Clearly

$$
\sum_{x \in A_{j}}|x|^{2} e^{-m|x|} \leqq \text { const } e^{-(m / 4) R 2 J}, \quad \text { for } j \geqq 1,
$$

and, by Theorem 1.2,

$$
P\left(\sim V_{N_{J}}\right)=1-P\left(V_{N_{j}}\right) \leqq C_{p} / N_{j}^{p},
$$


for any $p<\infty$, provided $|E|$ or $\delta$ are large enough. We now choose

$$
N_{j}=\frac{1}{8} R 2^{j} \text {. }
$$

It follows from (1.25)-(1.27) that

$$
\begin{aligned}
\varrho(E) D(E) \leqq & \lim _{\varepsilon \downarrow 0} \text { const }\left\{\varepsilon^{2}\left(e^{(m 8) R} R^{v+2}+\sum_{j=1}^{\prime} e^{-(m 8) R 2 \prime}\right)\right. \\
& \left.+\sum_{j=0}^{\infty} c_{v}\left(R 2^{j}\right)^{v+2-p} C_{p} 8^{p}\right\} .
\end{aligned}
$$

If we choose $p>v+2$, let $\varepsilon$ tend to 0 and $R$ tend to $x$ we conclude that $\varrho(E) D(E)=0$. Since $\varrho(E)>0$, for $E \in \operatorname{spec} H$, see (1.13), it follows that $D(E)=0$.

In the proof of Theorem 1.2 we shall take advantage of the fact that it is really enough to prove inequality (1.22) for the Green's function of the Hamiltonian in a finite volume. Let $\Lambda$ be some finite sublattice, and let $G_{\Lambda}(z, v ; x, y)$ be the Green's function of the Hamiltonian $H_{A}=-\Delta_{A}+v \chi_{\Lambda}$. In Appendix B we show that, for all $\varepsilon \neq 0$,

$$
\left|G_{\Lambda}(E+i \varepsilon, v ; x, y)\right| \leqq C_{\varepsilon} e^{-m(\varepsilon)|x-y|}
$$

for some constant $C_{\varepsilon}$ which is finite for $\varepsilon \neq 0$, and some $m(\varepsilon)$ proportional to $\varepsilon$, both independent of $v$ and $\Lambda$. From this and the resolvent equation it follows that, for $\varepsilon \neq 0$,

$$
\lim _{\Lambda \uparrow \mathbb{Z}^{v}} G_{\Lambda}(E+i \varepsilon, v ; x, y)=G(E+i \varepsilon, v ; x, y),
$$

uniformly for $x$ in finite subsets of $\mathbb{Z}^{v}$.

Theorem 1.2 follows from (1.29) and the following result, (see Appendix B for some details):

Theorem 1.2. There are constants $\lambda(p, m)$ and $E(\gamma, p, m)$ such that, for $m>0$ and $p<\infty$,

$$
\operatorname{Prob}\left\{v:\left|G_{\Lambda}(E+i \varepsilon, v ; 0, x)\right| \leqq e^{m(N-|x|)}, \varepsilon \neq 0\right\} \geqq 1-K_{p} / N^{p},
$$

uniformly in $\varepsilon \neq 0$ and in $\Lambda$, for some finite constant $K_{p}$, provided $\delta^{-1} \leqq \lambda(p, m)$, or $|E-2 v| \geqq E(i, p, m)$.

The proof of Theorem $1.2_{A}$ is the main goal of our paper.

The organization of our paper is as follows:

In Sect. 2 we sketch the basic strategy of the proof of Theorem $1.2_{\Lambda}$ and introduce an inductive scheme, extending over a sequence of increasing length scales, on which that proof is based. We reduce the proof of Theorem $1.2_{A}$ to some estimates on the Green's function of $H_{A}=-\Delta_{A}+v \chi_{A}$, where $A$ is an arbitrary finite sublattice having certain properties. Those estimates hold under inductively defined conditions on $v$. Furthermore, we state estimates on the probability that a potential $v$ satisfies those conditions.

In Sect. 3 we prove our basic (deterministic) estimates on finite volume Green's functions. Sections 2 and 3 really form the core of this paper. 
In Sect. 4 we prove some purely combinatorial results concerning subsets of $\mathbb{Z}^{v}$ which we shall need in our probability estimates. The results and the ideas of the proofs are closely related to results and arguments which appeared in $[7,8]$.

The basic probability estimates are stated and proven in Sect. 5, and in Sect. 6 the proof of Theorem $1.2_{\Lambda}$ is completed.

Some auxiliary results are collected in several appendices.

\section{An Outline of the Basic Strategy}

In this section we develop and inductive method which permits us to prove results concerning the decay of the Green's function in a finite volume $\Lambda$, for a class of "nonsingular" potentials which will turn out to have $d P$-measure close to 1 .

We choose an energy $E$, to be kept fixed in the following, and define a sequence of singular sets $S_{j}=S_{j}(E ; v)$ contained in $\mathbb{Z}^{v}$ which depend on $E$ and on the choice of the potential $v$. The sets $S_{j}$ have the property that

$$
S_{0} \supseteqq S_{1} \supseteqq S_{2} \supseteqq \ldots,
$$

with $\bigcap_{j} S_{j}=S_{\infty}=\emptyset$ with probability one.

The set of singular sites $S_{0}$ is defined by

$$
S_{0}=\left\{x \in \mathbb{Z}^{v}:|v(x)-E+2 v| \leqq N(E, \delta)\right\},
$$

where $N(E, \delta)=\frac{|E|}{2}$ when $d \lambda$ is gaussian and $|E|$ is large, and $N(E, \delta)=\delta^{1 / 2}$ whenever the disorder $\delta$ given by (1.21) is large. The sets $S_{k}, k=1,2, \ldots$, will be defined inductively.

Let $G_{A}(E ; x, y)$ denote the Green's function of $H_{A}=-\Delta_{A}+v \chi_{A}$, where $\Delta_{A}$ is the finite difference Laplacian with 0 Dirichlet boundary conditions on $\partial A$. We set

$$
G_{A}(E ; x, y)=0 \text {, unless } x \text { and } y \text { belong to } A \text {. }
$$

It is easy to show that if $A \cap S_{0}=\emptyset$ and $N(E, \delta)>2 v$ then

$$
\left|G_{A}(E+i \varepsilon ; x, y)\right| \leqq e^{-m(E)|x-y|}, \quad \forall \varepsilon \in \mathbb{R},
$$

where $m(E) \geqq \ln (N / 2 v), x, y$ in $A$, (see Appendix A). As $i$ increases, $S_{i}$ consists of increasingly singular points (sites) of $v$, in the sense that eigenvalues of "block" Hamiltonians, associated with certain subsets of $S_{i}$, are permitted to get closer to $E$. In Sect. 6 we prove that the probability that $S_{i}$ intersect some fixed, finite region goes to 0 rapidly with $i$.

The main purpose of this section is to give a precise definition of $S_{i}$, $i=0,1,2, \ldots$, and to state decay estimates for the Green's function $G_{A}(E ; x, y)$, assuming that $A \cap S_{k}=\emptyset$, for some $k<\infty$. We then present an estimate on the probability of choosing a potential $v$ with the property that for a given subset $A$ and an integer $k, A \cap S_{k}(E ; v)=\emptyset$. These results suffice to prove our main result, Theorem $1.2_{\Lambda}$.

We now present our inductive definition of $S_{k}, k \geqq 1$ : Assume that

$$
S_{0} \supseteqq S_{1} \supseteqq S_{2} \supseteqq \ldots \supseteqq S_{i}
$$


have already been defined, with $S_{0}$ given by (2.2). We define

$$
S_{i+1} \equiv S_{i} \sim S_{i}^{g}, \quad(\text { with } A \sim B \equiv A \backslash B),
$$

where

$$
S_{i}^{g} \equiv \bigcup_{\alpha} C_{i}^{\alpha} \subseteq S_{i}
$$

is the subset of "gentle singular sites of strength $i$ " of $S_{i}$. $S_{i}^{g}$ is a maximal union of components $C_{i}^{\alpha}$ satisfying the following condition.

Condition $A(i)$.

$$
\begin{gathered}
\operatorname{diam} C_{i}^{\alpha} \leqq d_{i} \equiv \exp \left[K_{0}(5 / 4)^{i}\right] \equiv r_{i-1} ; \\
\operatorname{dist}\left(C_{i}^{\alpha}, S_{i} \sim C_{i}^{\alpha}\right) \geqq 2 r_{i} \equiv 2 d_{i}^{5 / 4} ; \\
\operatorname{dist}\left(\operatorname{spec} H_{\overline{C_{1}^{\alpha}}}, E\right) \geqq e^{-\sqrt{d_{i}}},
\end{gathered}
$$

for all $\alpha$.

Here $\operatorname{spec} H_{D}$ is the set of eigenvalues of the "block Hamiltonian" $H_{D}=-\Delta_{D}+v \chi_{D}$, (D some finite region), and the set $\overline{C_{i}^{\alpha}}$ is defined by

$$
\overline{C_{i}^{\alpha}} \equiv\left\{x: \operatorname{dist}\left(x, C_{i}^{\alpha}\right) \leqq 4 d_{i}\right\} .
$$

In Condition $\mathrm{A}(\mathrm{i})$ and in the following, $\operatorname{diam} C$ is the diameter of $C \subset \mathbb{Z}^{v}$, and

$$
\operatorname{dist}(A, B) \equiv \inf |a-b|, \quad a \in A, b \in B,
$$

and $|\cdot|$ is the Euclidean distance.

Definition. A set $A$ is $k$-admissible iff

$$
\partial A \cap \overline{C_{j}^{\alpha}}=\emptyset, \quad j=0,1, \ldots, k .
$$

A set is admissible iff it is $k$-admissible, for all $k$.

We now describe some ideas underlying our analysis of the Green's function, $G_{A}(z ; x, y)$, where $A$ is some $k$-admissible subset of $\mathbb{Z}^{v}, k=0,1,2, \ldots$ Our estimates are based upon quantum mechanical perturbation theory, where the perturbations are chosen to be the operators which couple components of sets of singular points to their complement in $A$. In order to give a more precise description of our perturbation expansion we establish some more notation: Let $B$ be some subset of $A$, and let $\gamma \equiv \partial B$ be the set of links (nearest neighbor pairs) connecting sites in $B$ to sites in $\sim B$. We assume that $\gamma \subset A$. We define $\Gamma$ to be the operator corresponding to $\gamma$, whose matrix elements are given by

$$
\Gamma(i, j)= \begin{cases}1, & (i, j) \in \gamma \quad(|i-j|=1) \\ 0, & \text { otherwise } .\end{cases}
$$

Note that the Hilbert space, $l_{2}(A)$, associated with $A$, is a direct sum

$$
l_{2}(A)=l_{2}(B) \oplus l_{2}(A \sim B),
$$

and, correspondingly, the Hamiltonian $H_{A}$ is given by

$$
H_{A}=H_{B} \oplus H_{A \sim B}-\Gamma \text {. }
$$


Thus $\Gamma$ is the operator coupling the system in $B$ to the system in $A \sim B$. We define

$$
G_{B / A} \equiv G_{B} \oplus G_{A \sim B} .
$$

The first resolvent identity says that

$$
G_{A}=G_{B / A}+G_{B / A} \Gamma G_{A},
$$

or

$$
G_{A}(z ; x, y)=G_{B / A}(z ; x, y)+\sum_{\left(j, j^{\prime}\right) \in \gamma} G_{B / A}(z ; x, j) G_{A}\left(z ; j^{\prime}, y\right) .
$$

Our perturbation expansion is generated by iterating this identity, using different decompositions of $A$ which depend on $v$. It will be quite important to note that if $x \in B$,

$$
G_{B / A}(z ; x, j)=G_{B}(z ; x, j),
$$

and $G_{B}(z ; x, j)$ vanishes unless $j \in B$; furthermore $G_{B / A}(z ; x, y)=0$, unless $x$ and $y$ both belong to $B$ or to $A \sim B$, etc.

In the next section we prove the following decay estimate on the Green's function by iterated use of the resolvent identity.

Theorem 2.1. For $|E-2 v|+\delta$ sufficiently large and arbitrary real $\varepsilon$ there is a constant $m=m(E, \delta)>0$ such that if $A$ is a $k$-admissible subset of the lattice $\mathbb{Z}^{v}$, and $A \cap S_{k+1}=\emptyset$ then

$$
\left|G_{A}(E+i \varepsilon ; x, y)\right| \leqq \exp [-m|x-y|],
$$

provided $|x-y| \geqq \frac{1}{5} r_{k} \equiv \frac{1}{5} d_{k+1}$. The constant $m(E, \delta)$ is independent of $k$ and $A$. Moreover

$$
m(E, \delta) \geqq \frac{1}{2} \ln \{N(E, \delta)\}
$$

for $|E-2 v|+\delta$ sufficiently large. [Recall $N(E, \delta)$ is defined af ter Eq. (2.2).]

Note that this result which is proven in Sect. 3 is purely deterministic, i.e. it holds for each potential $v$ with the property that $A$ is $k$-admissible and $S_{k+1}(E ; v) \cap A=\emptyset$.

Our next task will be to estimate the $d P$-measure of the set of potentials, $W_{A, k}$. defined by

$$
W_{A, k} \equiv\left\{v: A \cap S_{k}(E ; v)=\emptyset\right\}
$$

In (2.14) the potential $v$ ought to be chosen in such a way that $A$ is $k$-admissible, and we are really interested in estimating the conditional probability

$$
\operatorname{Prob}\left(W_{A . k} \mid A \text { is } k \text {-admissible }\right) \text {. }
$$

This conditional probability can be estimated in terms of $P\left(W_{A . h}\right)$, for arbitrary, but $v$-independent subsets $A$. Moreover, bounding $P\left(W_{A . k}\right)$, for $v$-independent subsets $A$, can be reduced to estimating the probability that a component of $S_{j}^{g}(E ; v)$ in the sense of Condition $\mathrm{A}(\mathrm{j})$ contains a given site $x$ of the lattice, e.g. the origin; $(j \geqq k)$. Thus, let

$$
V_{x, j} \equiv\left\{v: S_{j}^{g}(E ; v) \ni x\right\}
$$


In Sects. 4 and 5 we shall prove combinatorial and probabilistic results which yield the following theorem, proven in Sect. 6.

Theorem 2.2. For large disorder $\delta$, or - if $d \lambda$ is the Gaussian, given by (1.14) - for large $|E-2 v|$, there exists a positive constant $k(E, \delta)$, with $k(E, \delta) \rightarrow+\infty$, as $\delta \rightarrow \infty$ or $|E| \rightarrow \infty$, such that

$$
\begin{aligned}
\operatorname{Prob}\left(V_{x, j}\right) & \equiv \int_{V_{x, j}} d P(v) \\
& \leqq \exp \left[-k(E, \delta) \ln d_{j-1}\right] \\
& =\exp \left[-k(E, \delta) K_{0}(5 / 4)^{j-1}\right] .
\end{aligned}
$$

Now suppose that the diameter of the region $A$ in Theorem 2.1 is bounded by

$$
\operatorname{diam} A \leqq r_{k}=\exp \left(K_{0}(5 / 4)^{k+1}\right) \text {. }
$$

Let $i \geqq k$. It follows from Condition A(i), (b) that at most one component of $S_{i}^{g}(E ; v)$ can intersect $A$. It then follows from Theorem 2.2 by straightforward arguments that

$$
\begin{aligned}
1-\operatorname{Prob}\left(W_{A, k}\right) & \leqq \sum_{j=k}^{\infty} \sum_{|x| \leqq r_{k}} \operatorname{Prob}\left(V_{x, j}\right) \\
& \leqq r_{k}^{v} \sum_{j=k}^{\infty} \exp \left[-k(E, \delta) K_{0}(5 / 4)^{j-1}\right] \\
& \leqq \exp \left[-\bar{k}(E, \delta) K_{0}(5 / 4)^{k}\right] \equiv d_{k}^{-\bar{k}(E, \delta)},
\end{aligned}
$$

by (2.16) and (2.17). Here $\bar{k}(E, \delta)$ is a constant which tends to $+\infty$, as $\delta$, or $|E|$, tend to $\infty$.

If we now combine Theorem 2.1 with Theorem 2.2 and (2.18) we arrive at the following basic probability estimate for the decay of the Green's function in a finite region. See Sects. 5 and 6 for a proof.

Theorem 2.3. Given any finite, positive number $p$, there exist constants $\lambda_{p}, E_{p}$ such that, for

$$
\delta^{-1}=\sup _{v}(d \lambda(v) / d v) \leqq \lambda_{p}, \quad \text { or } \quad|E-2 v| \geqq E_{p},
$$

the following event, $\mathscr{E}_{l}$, holds with probability at least $1-l^{-p}$ :

$\mathscr{E}_{l}$ is the set of all potentials $v$ with the property that there exists an admissible region $A$ such that $0 \in A$ and

$$
l / 2 \leqq \min _{b \in \partial A} \operatorname{dist}(0, b) \leqq \max _{b \in \partial A} \operatorname{dist}(0, b) \leqq l
$$

and

$$
\left|G_{A}(E+i \varepsilon, v ; x, y)\right| \leqq e^{-m|x-y|},
$$

provided $x$ and $y$ are in $A$, and $|x-y| \geqq l^{3 / 4}$. The decay rate $m=m(E, \delta)$ is as in Theorem 2.1.

Remark. The constraint $|x-y| \geqq l^{3 / 4}$ can be replaced by $|x-y| \geqq l^{\sigma}$, for arbitrary $\sigma>0$. 
In the proof of Theorem 2.3 (see Sects. 5 and 6) and in the considerations below we also use the following elementary lemma which is proven by using bounds on the density of states; see Appendix C (and Appendix A for additional information).

Lemma 2.4. Let $d \lambda(v) / d v$ be bounded. Then, for an arbitrary finite subset, $\Lambda$, of $\mathbb{Z}^{v}$ and an arbitrary $\kappa \leqq 1$,

$$
\operatorname{Prob}\left\{v: \operatorname{dist}\left(\operatorname{spec} H_{\Lambda}(v), E\right) \leqq \kappa\right\} \leqq \sqrt{2 \kappa} \varrho(E, \delta)|\Lambda|,
$$

where $\bar{\varrho}(E, \delta) \rightarrow 0$, as $|E|$, or $\delta$ tend to $+\infty$.

The end of this section is devoted to proving that Theorem 2.3 and Lemma 2.4 imply our main result, Theorem $1.2_{\Lambda}$ (Sect. 1):

First, we assume that

$$
|x| \geqq N / 2 \text {, }
$$

where $N$ is the positive number specified in Theorem $1.2_{\Lambda}$. Let $\left(A_{j}\right)_{j=0,1,2, \ldots}$ be a sequence of admissible sets for which Theorem 2.3 holds, with

$$
l=l_{j} \equiv 2|x| \cdot 4^{j} .
$$

We set $\gamma_{j} \equiv \partial A_{j}$, and let $\Gamma_{j}$, be the corresponding boundary operator coupling the systems in $A_{j}$ and in $\sim A_{j}$. To simplify notation we set

$$
G \equiv G_{A}, \quad G_{j} \equiv G_{A_{j}} .
$$

[Note that, for $j$ large enough, $A_{j} \supseteqq \Lambda$, and all sites in $\sim \Lambda$ belong to $\sim S_{0}$, since $v(j)=+\infty$, for $j \in \sim \Lambda$.] By iterating the resolvent identity (2.13) we have

$$
G(E+i \varepsilon, v ; 0, x)=\left[G_{0}+G_{0} \Gamma_{0} G_{1}+G_{0} \Gamma_{0} G_{1} \Gamma_{1} G_{2}+\ldots\right](E+i \varepsilon, v ; 0, x) .
$$

Notice that the matrix elements of $G_{j}$ in the series on the right hand side of (2.21) are evaluated at sites which are separated by a distance of at least

$$
-2|x| 4^{j-1}+\frac{1}{2} 2|x| 4^{j} \geqq \frac{1}{2}|x| 4^{j} .
$$

By Theorem 2.3 we know that if $v \in \mathscr{E}_{l}$, then

$$
\left|G_{j}\left(E+i \varepsilon ; z, z^{\prime}\right)\right| \leqq \exp \left[-m(E, \delta)\left|z-z^{\prime}\right|\right],
$$

provided $\left|z-z^{\prime}\right| \geqq l_{j}^{3 / 4}=\left(2|x| 4^{j}\right)^{3 / 4}$. By (2.22), this condition is satisfied in all terms on the right hand side of (2.21). Thus, it is easy to see that the series on the right hand side of (2.21) converges, for every potential $v \in \bigcap_{j=0}^{\infty} \mathscr{E}_{l j}$, and is bounded by

$$
|G(E+i \varepsilon, v ; 0, x)| \leqq K e^{-m(E, \delta)|x|},
$$

for $|x| \geqq N / 2$, where $K$ is some constant independent of $E, N$ and of $v \in \bigcap_{j=0}^{\infty} \mathscr{E}_{l_{j}}$. We now estimate Prob $\left[\bigcap_{j=0}^{\infty} \mathscr{E}_{l_{j}}\right]$ : We choose an arbitrary, positive integer $p$ and require that

$$
\delta^{-1} \leqq \lambda_{p}, \quad \text { or } \quad|E-2 v| \geqq E_{p},
$$


where $\lambda_{p}$ and $E_{p}$ are the constants introduced in Theorem 2.3. Then

$$
\begin{aligned}
\operatorname{Prob}\left[\bigcap_{j=0}^{\infty} \mathscr{E}_{l_{j}}\right] & \equiv P\left[\bigcap_{j=0}^{\infty} \mathscr{E}_{l_{j}}\right] \\
& =1-P\left[\bigcup_{j}\left(\sim \mathscr{E}_{l_{j}}\right)\right] \\
& \geqq 1-\sum_{j} P\left[\sim \mathscr{E}_{l_{j}}\right] \\
& \geqq 1-\sum_{j} l_{j}^{-p} \\
& \geqq 1-\operatorname{const} l_{0}^{-p} \geqq 1-K_{p}^{\prime} N^{-p},
\end{aligned}
$$

and we have used (2.19) and (2.20) in the last inequality. This proves Theorem $1.2_{\Lambda}$ under the assumption that $|x| \geqq N / 2$.

If $|x|<N / 2$ we set $l_{j}=N \cdot 4^{j+1}, j=0,1,2, \ldots$ Let $\|\cdot\|$ denote the operator norm. By Lemma 2.4 we know that the inequalities

$$
\begin{aligned}
\left|G_{0}(E+i \varepsilon, v ; 0, x)\right| & \leqq\left\|G_{0}\right\| \leqq e^{m N / 2} \\
& \leqq e^{(N-|x|)}, \quad|x| \leqq \frac{N}{2},
\end{aligned}
$$

hold with probability

$$
1-2 e^{-m N / 4}\left|A_{0}\right| \bar{\varrho}(E, \delta) \geqq 1-2(8 N)^{v} e^{-m N / 4} \bar{\varrho}(E, \delta) .
$$

In (2.24) and (2.25) it is not required that $A_{0}$ be admissible, since in Lemma 2.4 it is not assumed that $\Lambda$ is admissible. (In fact, admissibility of a set $A$ is irrelevant, whenever one needs only a norm estimate on $G_{A}$.) We denote by $\mathscr{E}^{(0)}$ the event that inequality (2.24) holds. Thus by (2.25)

$$
P\left(\mathscr{E}^{(0)}\right) \geqq 1-2(8 N)^{\prime} e^{-m N / 4} \underline{\varrho}(E, \delta),
$$

with $m=m(E, \delta)$ as in Theorem 2.1 .

It is still assumed that the regions $A_{j}, j=1,2,3, \ldots$, are admissible. The resolvent expansion on the right hand side of (2.21) can now be estimated by using (2.24) and Theorem 2.3, provided the potential $v$ belongs to the subset

$$
\mathscr{E}^{\mathscr{E}(0)} \cap\left[\bigcap_{j=1}^{\infty} \mathscr{E}_{l_{j}}\right] .
$$

Furthermore

$$
\begin{aligned}
P\left(\mathscr{E}^{(0)} \cap\left[\bigcap_{j=1}^{\infty} \mathscr{E}_{l_{J}}\right]\right) & \geqq 1-P\left(\sim \mathscr{E}^{(0)}\right)-\sum_{j=1}^{\infty} P\left(\sim \mathscr{E}_{l_{J}}\right) \\
& \geqq 1-\frac{K_{p}^{\prime \prime}}{N^{p}}
\end{aligned}
$$

if $|E-2 v|$ or $\delta$ is large.

This completes the proof of Theorem $1.2_{\Lambda}$. 


\section{Decay Estimates on Green's Functions}

In this section we prove inductively a family of inequalities for $G_{A}(E+i \varepsilon, v ; x, y)$ indexed by distance scales $|x-y| \approx d_{k} \equiv \exp \left[K_{0}\left(\frac{5}{4}\right)^{k}\right]$, for admissible $A$ such that $A \cap S_{k}=\emptyset$. Let $\mathscr{I}_{k}$ indicate the following inequality:

$$
\left|G_{A}(E+i \varepsilon, v ; x, y)\right| \leqq e^{-m_{k}|x-y|},
$$

for $|x-y| \geqq \frac{1}{5} d_{k} \equiv \frac{1}{5} r_{k-1}$, assuming that $A$ is $(k-1)$-admissible, and

$$
A \cap S_{k}=\emptyset \text {. }
$$

The exponential decay rate ("mass") $m_{k}$ is given by

$$
m_{k}=\ln \left(\frac{N(E, \delta)}{2 v}\right)-\sum_{i=0}^{k} C d_{i}^{-(1 / 4)},
$$

for some finite constant $C$, with $N(E, \delta)$ as in (2.2).

We note that the sequence $\left(m_{k}\right)_{k=0}^{\infty}$ is decreasing and that if we choose $d_{0}=e^{K_{0}}$ large enough

$$
m \equiv \lim _{k \rightarrow \infty} m_{k} \geqq \frac{1}{2} \ln (N(E, \delta))>0,
$$

provided $|E|+\sqrt{\delta}$ is chosen sufficiently large.

It is quite easy to prove $\mathscr{I}_{0}$, see Appendix A. We shall prove $\mathscr{I}_{k}$ by induction. These inequalities together with (3.3) imply Theorem 2.1.

We now assume $\mathscr{I}_{1}, i=0,1, \ldots, k$ and propose to establish $\mathscr{I}_{k+1}$.

Since $A$ is chosen in such a way that

$$
A \cap S_{k+1}=A \cap\left(S_{k} \sim S_{k}^{g}\right)=\emptyset,
$$

we see that $\mathscr{I}_{k}$ holds for admissible subsets of

$$
A \sim\left(S_{k}^{g} \cap A\right) .
$$

We now consider those components $C_{k}^{\alpha}$ of $S_{k}^{g}$ which belong to $A$. By (3.4) and (3.5), these sets represent the only "new singularities" of the potential $v$ which might obstruct the validity of $\mathscr{I}_{k+1}$. [If $A \cap S_{k}^{g}=\emptyset$ then $A \cap S_{k}=\emptyset$, by (3.4), and $\mathscr{I}_{k+1}$ follows trivially from $\mathscr{I}_{k}$. $]$ We shall prove $\mathscr{I}_{k+1}$ by using $\mathscr{I}_{k}$ on $G_{A \sim\left(S_{k}^{g} \cap A\right)}(E+i \varepsilon ; x, y)$ and putting back the couplings to $S_{k}^{g} \cap A$ perturbatively. The convergence of our perturbative expansions can be controlled with the help of Condition $\mathrm{A}(\mathrm{k})$, Sect. 2, and our induction hypotheses.

We now start discussing the details of the proof. Let $R_{k}^{\alpha}$ be a $k$-admissible region containing a component $C_{k}^{\alpha}$ of $S_{k}^{g}(E ; v)$ and with the property that

and

$$
\left.\begin{array}{c}
\frac{1}{5} d_{k+1} \leqq \operatorname{diam} R_{k}^{\alpha} \leqq \frac{3}{2} d_{k+1}, \\
\operatorname{dist}\left(\partial R_{k}^{\alpha}, \overline{C_{k}^{\alpha}}\right)>0, \text { with } \overline{C_{k}^{\alpha}} \text { as in }(2.7) .
\end{array}\right\}
$$

Since, by Condition $\mathrm{A}(\mathrm{k})$, (b),

$$
\operatorname{dist}\left(C_{k}^{\alpha}, S_{k} \sim C_{k}^{\alpha}\right) \geqq 2 r_{k} \equiv 2 d_{k+1},
$$

[see (2.5)], it follows that

$$
R_{k}^{\alpha} \cap\left(S_{k} \sim C_{k}^{\alpha}\right)=\emptyset,
$$


in particular,

$$
R_{k}^{\alpha} \cap S_{k+1}=\emptyset,
$$

and $R_{k}^{\alpha}$ is an admissible set. The existence of regions $R_{k}^{\alpha}$ with the above properties is checked in Appendix D. Our first technical result in this section is

Lemma 3.1. Let $R \equiv R_{h}^{\alpha}$ be as above, and choose $d_{0}$ and $|E|+V \bar{\delta}$ sufficiently large, independently of $k$. If

then

$$
|x-y| \geqq \frac{1}{5} d_{k+1},
$$

where

$$
\left|G_{R}(E+i \varepsilon, v ; x, y)\right| \leqq \exp \left[-\left(m_{k}-\mu_{k}\right)|x-y|\right] \text {, }
$$

$$
\mu_{k}=45 d_{k}^{-1 / 4} m_{0} .
$$

Proof. Let $C \equiv C_{k}^{\alpha}, \bar{C} \equiv \overline{C_{k}^{\alpha}}[\operatorname{see}(2.7)]$, and let $B$ be a $(k-1)$-admissible set such that $R \sim B$ is $(k-1)$-admissible, as well, and

$$
\left.\begin{array}{l}
\bar{C} \supset B \supset C, \\
\operatorname{dist}(\partial B,\{x, y\}) \geqq d_{k} \\
\operatorname{dist}(\sim B, C) \leqq 3 d_{k} .
\end{array}\right\}
$$

Since $|x-y| \geqq \frac{1}{5} d_{k+1}$, at most one site, $x$ or $y$ may be contained in any one of the sets $C, B, \bar{C}$, while the other one is at a distance $\gg d_{k}$ from $\bar{C}$. The existence of a set $B$ with the above properties is verified in Appendix D. We set

$$
\bar{\gamma}=\partial \bar{C}, \quad \gamma=\partial B, \quad \text { and } \quad Q=R \sim B .
$$

The geometrical situation envisaged here is depicted in Fig. 1.

We define $\bar{\Gamma}$ and $\Gamma$ to be the operators corresponding to $\bar{\gamma}$ and $\gamma$, respectively [see (2.9)]. To prove (3.9) we now distinguish the following two cases:

(1) $x \in Q, y \in Q$; and

(2) $x \in B, y \in Q$,

[the case $y \in B, x \in Q$ is clearly analogous to (2)]. We first study (1): By using

Fig. 1




the resolvent identities (2.12) we obtain

$$
G_{R}(E+i \varepsilon ; x, y)=\left[G_{Q}+G_{Q} \Gamma G_{R} \Gamma G_{Q}\right](E+i \varepsilon ; x, y),
$$

since, for $x$ and $y$ in $Q$, the term

$$
\left[G_{Q} \Gamma G_{Q / R}\right](E+i \varepsilon ; x, y)
$$

vanishes ( $\Gamma$ couples $Q$ to $B$ !). Since $Q$ is $(k-1)$-admissible and $Q \cap S_{k}=\emptyset, \mathscr{I}_{k}$ implies

$$
\left|G_{Q}(E+i \varepsilon ; u, w)\right| \leqq \exp \left[-m_{k}|u-w|\right],
$$

provided $|u-w| \geqq \frac{1}{5} d_{k}$. By (3.10), inequality (3.12) can be applied to estimate $G_{Q}$ in both terms on the right side of (3.11). To complete our estimate of $\left|\left[G_{Q} \Gamma G_{R} \Gamma G_{Q}\right](E+i \varepsilon ; x, y)\right|$ we appeal to the following

Sub-Lemma 3.2. Let $u$ and $w$ belong to $B$. Then

$$
\left|G_{R}(E+i \varepsilon ; u, w)\right| \leqq 2 \exp \sqrt{d_{k}} .
$$

Assuming (3.13), and using (3.12), we see that Eq. (3.11) yields the bound

$$
\begin{aligned}
&\left|G_{R}(E+i \varepsilon ; x, y)\right| \leqq e^{-m_{k}|x-y|}+\sum_{\begin{array}{c}
\left(u, u^{\prime}\right) \in \gamma \\
\left(w, w^{\prime}\right) \in \gamma
\end{array}} e^{-m_{k}(|x-u|+|w-y|)} 2 e^{\sqrt{d_{k}}} \\
& \leqq e^{-m_{k}|x-y|}\left\{1+2|\gamma|^{2} e^{7 m_{k} d_{k}+\gamma \overline{d_{k}}}\right\}
\end{aligned}
$$

and we have used the fact that

$$
\left|u^{\prime}-w^{\prime}\right| \leqq \operatorname{diam} B \leqq 7 d_{k},
$$

by (3.10) and Condition $\mathrm{A}(\mathrm{k}),(\mathrm{a})$. In (3.14) $\left(u, u^{\prime}\right)$ and $\left(w, w^{\prime}\right)$ are bonds (nearest neighbor pairs) belonging to $\gamma=\partial B$, with $u, w \in Q$, and $|\gamma|$ is the area of $\gamma$. If we set

$$
\mu_{k}=\frac{9 d_{k} m_{0}}{(1 / 5) d_{k+1}}=45 d_{k}^{-1 / 4} m_{0},
$$

we see that, for $|x-y| \geqq \frac{1}{5} d_{k+1}$ and $d_{0},|E|+\sqrt{\delta}$ so large that $\sqrt{d_{k}} \leqq m_{k} d_{k}$,

$$
\left|G_{R}(E+i \varepsilon ; x, y)\right| \leqq \exp \left[-\left(m_{k}-\mu_{k}\right)|x-y|\right] .
$$

We now come to the discussion of case (2), i.e., $x \in B, y \in Q$ : We use the resolvent identity

$$
G_{R}=G_{Q / R}+G_{R} \Gamma G_{Q / R}
$$

which yields

$$
G_{R}(E+i \varepsilon ; x, y)=\left[G_{R} \Gamma G_{Q}\right](E+i \varepsilon ; x, y),
$$

because [see (2.11)]

$$
G_{Q / R}(E+i \varepsilon ; x, y)=0, \text { for } \quad x \in B \subset \sim Q, \quad y \in Q,
$$

and

$$
G_{Q / R}(E+i \varepsilon ; w, y)=G_{Q}(E+i \varepsilon ; w, y), \quad \text { for } \quad y \in Q \text {. }
$$


Hence, using Sub-Lemma 3.2 to bound $\left|G_{R}(E+i \varepsilon ; x, u)\right|, x, u$ in $B$, and $\mathscr{I}_{k}$ to bound $\left|G_{Q}\left(E+i \varepsilon ; u^{\prime}, y\right)\right|$ (which is possible, since

$$
\left|y-u^{\prime}\right| \geqq|y-x|-\operatorname{diam} B \geqq \frac{1}{5} d_{k+1}-7 d_{k}>\frac{1}{5} d_{k},
$$

for $d_{0}$ large enough), we obtain

$$
\begin{aligned}
\left|G_{R}(E+i \varepsilon ; x, y)\right| & \leqq \sum_{\left(u, u^{\prime}\right) \in \gamma}\left|G_{R}(E+i \varepsilon ; x, u)\right|\left|G_{Q}\left(E+i \varepsilon ; u^{\prime}, y\right)\right| \\
& \leqq 2|\gamma| e^{\sqrt{d_{k}}} e^{-m_{k}\left(|x-y|-7 d_{k}\right)} \\
& \leqq \exp \left[-\left(m_{k}-\mu_{k}\right)|x-y|\right] .
\end{aligned}
$$

This completes the proof of Lemma 3.1.

We now turn to our

Proof of Sub-Lemma 3.2. By alternated application of the two resolvent identities

$$
\begin{aligned}
G_{R} & =G_{\bar{C} / R}+G_{\bar{C} / R} \bar{\Gamma} G_{R} \\
& =G_{B / R}+G_{B / R} \Gamma G_{R}
\end{aligned}
$$

we obtain the expansion

$$
G_{R}(E+i \varepsilon ; u, w)=\left[G_{\bar{C}}+G_{\bar{C}} \bar{\Gamma} G_{B / R}+G_{\bar{C}} \bar{\Gamma} G_{B / R} \Gamma G_{\bar{C}}+\ldots\right](E+i \varepsilon ; u, w) .
$$

Note that since $\Gamma$ and $u, w$ are all contained in $\bar{C}, G_{\bar{C} / R}=G_{\bar{C}}$, in all terms contributing to the right side of (3.15). Factors $G_{\bar{C}}$ on the right side of (3.15) are estimated by using the norm bound

$$
\left|G_{\bar{C}}(E+i \varepsilon ; u, w)\right| \leqq\left\|G_{\bar{C}}(E+i \varepsilon ; \cdot, \cdot)\right\| \leqq e^{\sqrt{d_{k}}},
$$

for $u$ and $w$ in $\bar{C}$. This bound follows from Condition $\mathrm{A}(\mathrm{k}),(\mathrm{c})$, see (2.6), i.e.,

$$
\operatorname{dist}\left(\operatorname{spec} H_{\bar{C}}, E\right) \geqq e^{-\sqrt{d_{k}}} \text {. }
$$

Next, we observe that, in all non-vanishing terms on the right side of (3.15), we have

$$
G_{B / R}=G_{R \sim B} \equiv G_{Q},
$$

since $\bar{\Gamma} \subset Q$. These factors can be estimated by using the induction hypothesis $\mathscr{I}_{k}$, since

$$
\operatorname{dist}(\bar{\gamma}, \gamma) \geqq d_{k}>\frac{1}{5} d_{k}
$$

and $(R \sim B) \cap S_{k}=\emptyset$. [To show (3.17) we recall that, by (2.7) and (3.10),

$$
\left.\operatorname{dist}(\bar{\gamma}, \gamma) \geqq 4 d_{k}-\operatorname{dist}(\sim B, C) \geqq d_{k} \cdot\right]
$$

Thus, applying $\mathscr{I}_{k}$ to $G_{Q}$ we find

$$
\left|\left(\bar{\Gamma} G_{Q} \Gamma\right)\left(z, z^{\prime}\right)\right| \leqq e^{-m_{k}\left|z-z^{\prime}\right|} .
$$

By (3.16) and (3.18), the series on the right side of (3.15) can be bounded by

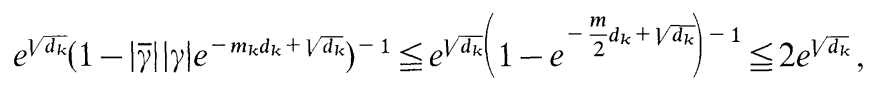


if $|E|+\sqrt{\delta}$ is large enough and $d_{0}$ is chosen sufficiently large. This completes the proof of Sub-Lemma 3.2, and hence of Lemma 3.1.

We now use Lemma 3.1 to complete the induction step,

$$
\mathscr{I}_{k} \text { implies } \mathscr{I}_{k+1}
$$

Thus, let $A$ be some $k$-admissible region, with

$$
A \cap S_{k+1}=\emptyset \text {. }
$$

We may also assume that

$$
\operatorname{diam} A \geqq \frac{1}{5} d_{k+1} ;
$$

otherwise $\mathscr{I}_{k+1}$ is empty [see (3.1)]. Furthermore we may assume that

$$
A \cap S_{k}^{g} \neq \emptyset,
$$

since otherwise $\mathscr{I}_{k+1}$ follows trivially from $\mathscr{I}_{k}$, as already remarked. If

$$
\operatorname{diam} A \leqq \frac{3}{2} d_{k+1}
$$

$\mathscr{I}_{k+1}$ follows directly from Lemma 3.1, by setting $R=A$ in Lemma 3.1 [see (3.7)]. Thus we may assume henceforth that

$$
\operatorname{diam} A>\frac{3}{2} d_{k+1} \text {. }
$$

To establish $\mathscr{I}_{k+1}$, we shall combine Lemma 3.1 with a method closely related to one used in [13] to prove exponential clustering of spin-spin correlations in statistical mechanics. We need the following easy geometrical result which is proven in Appendix D.

Lemma 3.3. If $d_{0}$ is chosen sufficiently large then, given any site $p \in A$, there is a $k$-admissible region $R_{p} \subset A$ such that

$$
\begin{gathered}
\operatorname{dist}\left(p, \partial R_{p} \sim \partial A\right) \geqq \frac{1}{2} d_{k+1}, \\
\operatorname{diam} R_{p} \leqq \frac{3}{2} d_{k+1},
\end{gathered}
$$

and

$$
\operatorname{dist}\left(\{x, y\}, \partial R_{p}\right) \geqq \frac{1}{5} d_{k+1},
$$

where $x$ and $y$ are two sites in $A$, with $|x-y| \geqq \frac{1}{5} d_{k+1}$.

Let $\gamma_{p}=\partial R_{p}$ and let $\Gamma_{p}$ be the corresponding operator (coupling $R_{p}$ and $\sim R_{p}$ ). We shall iterate the resolvent identity

$$
\begin{aligned}
G_{A}(E+i \varepsilon ; p, y)= & {\left[G_{R_{p}}+G_{R_{p}} \Gamma_{p} G_{A}\right](E+i \varepsilon ; p, y) } \\
= & G_{R_{p}}(E+i \varepsilon ; p, y)+\sum_{\left(u, u^{\prime}\right) \in \gamma_{p}} G_{R_{p}}(E+i \varepsilon ; p, u) \\
& \cdot G_{A}\left(E+i \varepsilon ; u^{\prime}, y\right) .
\end{aligned}
$$

(See Fig. 2 for a graphical representation of a typical term.)

In order to prove $\mathscr{I}_{k+1}$ [see (3.1) and (3.2)], we begin by setting $p=x$. The factor $G_{A}\left(E+i \varepsilon ; u^{\prime}, y\right)$ on the right side of (3.23) is expanded by iterating (3.23) with $p=u^{\prime}$, etc. This process is repeated infinitely often. The resulting series expansion is 
Fig. 2

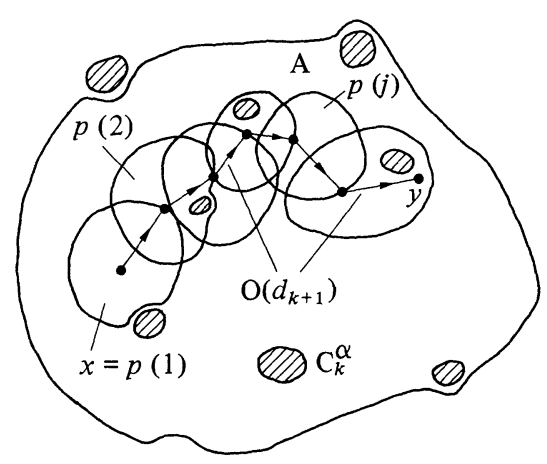

rapidly convergent, and the remainder term, after $n$ iterations, tends to 0 , as $n \rightarrow x$, whenever $\varepsilon \neq 0$, because the matrix elements of all Greens' functions $G_{R_{p(j)}}(E+i \varepsilon ; p(j), p(j+1)), j=1,2, \ldots$, appearing in the iteration of (3.23), are evaluated at sites which are separated by a distance of at least $\frac{1}{5} d_{k+1}$. If $R_{p(j)} \cap S_{k}=\emptyset$ then the bound

$$
\left|G_{R_{p(j)}}(E+i \varepsilon ; p(j), p(j+1))\right| \leqq e^{-m_{k}^{\prime}|p(j)-p(j+1)|},
$$

with $m_{k}^{\prime}=m_{k}$, follows directly from $\mathscr{I}_{k}$. If $R_{p(j)}$ contains a component $C_{k}^{\alpha}$ of $S_{k}^{g}$ then (3.24) follows from Lemma 3.1 with $R=R_{p(j)}$, and $m_{k}^{\prime}=m_{k}-\mu_{k}$ (where $\mu_{k}$ is as in Lemma 3.1).

In order to estimate the sum of products of these Green's functions, let

$$
m_{k}^{\prime \prime} \equiv m_{k}-\mu_{k} .
$$

Using (3.24), we bound the $n^{\text {th }}$ term in our expansion by

$$
\sum_{\{p(2), \ldots, p(n)\}} \prod_{j=1}^{n} e^{-m_{k}^{\prime \prime}|p(j)-p(j+1)|}
$$

with $p(1)=x, p(n+1)=y$. Setting

$$
l_{j} \equiv|p(j)-p(j+1)|
$$

$l_{1}, \ldots, l_{n}$ are constrained by the following conditions:

$$
\sum_{j=1}^{n} l_{j} \equiv l \geqq|x-y| \geqq \frac{1}{5} d_{k+1},
$$

and

$$
\frac{3}{2} d_{k+1} \geqq l_{j} \geqq \frac{1}{2} d_{k+1}, \quad \text { for } \quad j=1,2, \ldots, n-1 .
$$

These conditions follow from (3.23), and from (3.21) and (3.22). We note that, by (3.26) and (3.27),

$$
n \leqq\left(2 l / d_{k+1}\right)+1 \leqq 7 l / d_{k+1}
$$


By summing (3.25) over all values of $n$ and taking (3.26)-(3.28) into account, we obtain the following upper bound for the expansion resulting from our iterations of (3.23):

$$
\begin{aligned}
\left|G_{A}(E+i \varepsilon ; x, y)\right| & \sum_{l \geqq|x-y|} e^{-m_{k}^{\prime \prime} l}\left(\text { const } d_{k+1}\right)^{7 v l l d_{k+1}} \\
& \leqq \exp \left[-m_{k+1}|x-y|\right]
\end{aligned}
$$

with

$$
\begin{aligned}
m_{k+1} & \equiv m_{k}^{\prime \prime}-7 v \ln \left(\text { const } d_{k+1}\right) / d_{k+1} \\
& \geqq m_{k}-50 m_{0} d_{k+1}^{-1 / 4},
\end{aligned}
$$

provided $d_{0}$ is chosen sufficiently large. In (3.29) it is assumed, implicitly, that the remainder term, after $n$ iterations of (3.23), tends to 0 , as $n \rightarrow \infty$. That remainder term can be bounded by

$$
\left[\sum_{\{p(2), \ldots, p(n)\}} \prod_{j=1}^{n} \mathrm{e}^{-m_{k}^{\prime \prime}|p(j)-p(j+1)|}\right]\left\|G_{A}\right\| \leqq\left(\text { const } d_{k+1} e^{-m_{k}^{\prime \prime} d_{k+1} / 2}\right)^{n} \varepsilon^{-1}
$$

which tends to 0 , as $n \rightarrow \infty$, for arbitrary $\varepsilon \neq 0$, provided $d_{0}$ is chosen sufficiently large.

To summarize, we have proven that for every $k$-admissible region $A$ with $A \cap S_{k+1}=\emptyset$ and arbitrary sites $x, y$ in $A$ with the property that $|x-y| \geqq \frac{1}{5} d_{k+1}$

with

$$
\left.\begin{array}{l}
\left|G_{A}(E+i \varepsilon ; x, y)\right| \leqq e^{-m_{k+1}|x-y|} \\
m_{k+1} \geqq m_{k}-50 m_{0} d_{k}^{-1 / 4}
\end{array}\right\}
$$

Thus $\mathscr{I}_{k+1}$ holds [see (3.1) and (3.2)], and the induction step is finished. The proof of Theorem 2.1 is therefore complete.

Remark. The condition that $A$ and $R_{p}$ be admissible regions is important, because $\partial R_{p}$ and $\partial A$ are required not to intersect $S_{k}^{g}$ for Lemma 3.1 to apply.

In the remaining sections we estimate the probability of the event that

$$
A \cap S_{k}=\emptyset,
$$

where $S_{k}=S_{k}(E ; v)$ depends on the potential $v$. This will yield Theorems 2.2 and 2.3.

\section{Entropy - Combinatorial Bounds}

In this section we establish some general combinatorial results which provide estimates on the number of subsets $D \subset \mathbb{Z}^{v}$ which contain the origin and have prescribed "volumes," in a sense that we shall make precise. These results play an important role in the proof of our basic probability estimates stated in Theorem 2.3 (see Sect. 6). Let $\mathbb{Z}^{v}(n) \equiv 2^{n} \mathbb{Z}^{v}, n \geqq 0$. With each site $k \in \mathbb{Z}^{v}(n-1)$ we associate a cube $c_{n}(k)$ with sides of length $2^{n}$ parallel to the lattice axes and centered at $k$. Each such cube is called an $n$-cube. [For $n=0, \mathbb{Z}^{v}(-1) \equiv \mathbb{Z}^{v}$, and a 0 -cube is a site in $\mathbb{Z}^{v}$.] We note that two adjacent $n$ cubes overlap in a rectangular region of width $2^{n-1}$, for $n \geqq 1$. Using this fact it is easy to show 
Proposition 4.1. Every finite subset $D \subset \mathbb{Z}^{v}$ is entirely contained in a single $n$-cube, $c_{n}$, provided that $2^{n} \geqq 2 \operatorname{diam} D$.

By $\mathscr{C}_{n}$ we denote an arbitrary collection of $n$-cubes and define $\left|\mathscr{C}_{n}\right|$ to be the cardinality of $\mathscr{C}_{n}$. Given a subset $D \subset \mathbb{Z}^{v}$ we define $\mathscr{C}_{n}(D)$ to be a minimal family of $n$-cubes $\left[\right.$ i.e. $\left|\mathscr{C}_{n}(D)\right|$ is minimal] which cover $D$. We define the "volume" of $D$ on scale $2^{n}$ to be

and the total volume

$$
V_{n}(D) \equiv\left|\mathscr{C}_{n}(D)\right|,
$$

$$
V(D)=\sum_{n=0}^{n_{0}(D)} V_{n}(D),
$$

where $n_{0}(D)$ is the smallest integer such that $2^{n_{0}(D)} \geqq 2 \operatorname{diam} D$.

Theorem 4.2. If $N(V)$ is the number of subsets $D \subset \mathbb{Z}^{v}$ such that $V(D)=V$ and $0 \in D$ then

$$
N(V) \leqq 3^{v} 2^{V}\left(2 \cdot 3^{v}\right)^{V} \leqq e^{K_{v} V},
$$

for some constant $K_{v}$.

Remark. Note $\ln N(V)$ is related to what in physics is called entropy.

To prove this theorem, let us first consider the family $\mathscr{F}$ of sets $D$ containing the origin with specified $n$-volumes: $V_{n}(D)=V_{n}$. Let $N\left(\left\{V_{n}\right\}\right)$ be the cardinality of $\mathscr{F}$. Now consider a collection $\mathscr{C}_{n}$ of $n$-cubes such that $\left|\mathscr{C}_{n}\right|=V_{n}$. We think of $\mathscr{C}_{n}$ as specifying our set $D$ "on scale $2^{n}$." Let $N\left(\mathscr{C}_{n}, V_{n-1}\right)$ be the number of covers $\mathscr{C}_{n-1}^{\alpha}$ subordinate to $\mathscr{C}_{n}$ (i.e. each cube of $\mathscr{C}_{n-1}^{\alpha}$ is covered by some cube in $\mathscr{C}_{n}$ ) such that $\left|\mathscr{C}_{n-1}^{\alpha}\right|=V_{n-1}, \alpha=1,2, \ldots, N\left(\mathscr{C}_{n}, V_{n-1}\right)$.

\section{Proposition 4.3.}

$$
N\left(\mathscr{C}_{n}, V_{n-1}\right) \leqq\left(2 \cdot 3^{v}\right)^{V_{n-1}}
$$

This bound clearly implies that

$$
N\left(\left\{V_{n}\right\}\right) \leqq 3^{v}\left(2 \cdot 3^{v}\right)^{V}
$$

where the factor $3^{v}$ arises from counting the number of covers $\mathscr{C}_{n_{0}},\left(n_{0}=n_{0}(D)\right.$,) consisting of a single $n_{0}$-cube which encloses the origin. Since there are less than $2^{V}$ choices of sequences $\left(V_{n}\right), n=0,1,2, \ldots$ for which $\sum V_{n}=V$, Theorem 4.1 follows.

Proof of Proposition 4.3. Given a cube $c \in \mathscr{C}_{n}$ and a collection $\mathscr{C}_{n-1}^{\alpha}$ as specified above, let $N_{c}^{\alpha}$ be the number of $(n-1)$-cubes in $\mathscr{C}_{n-1}^{\alpha}$ contained in $c$. Clearly

$$
\sum_{c \in \mathscr{C}_{n}} N_{c}^{\alpha}=V_{n-1}
$$

We now ask how many collections $\mathscr{C}_{n-1}^{\alpha}$ are there for which $N_{c}^{\alpha}=N_{c}$ with $\sum N_{c}=V_{n-1}$. Note that there are $3^{v}$ possible positions of an $(n-1)$-cube inside some arbitrary $n$-cube. Thus the answer to the question posed above is that the number of collections with specified $N_{c}^{\alpha}=N_{c}$ is bounded by

$$
\prod_{c \in \mathscr{C}_{n}}\left(\begin{array}{c}
3^{v} \\
\mathrm{~N}_{c}
\end{array}\right) \leqq 3^{v V_{n-1}} .
$$

Now summing over the $2^{V_{n-1}}$ choices of $\left\{N_{c}\right\}_{c \in \mathscr{C}_{n}}$ we see that (4.4) follows. 
Remark. These bounds are very crude and can be improved with more effort. This might be important in other applications, e.g. to expansions in statistical mechanics.

In order to obtain probability estimates for the components $D \equiv C_{i}^{\alpha}$ of sets of singular sites, introduced in Sects. 2 and 3 , it is convenient to define a family $\mathscr{C}_{n}^{\prime}(D)$ of "isolated" cubes. [Cubes in $\mathscr{C}_{n}^{\prime}(D)$ will turn out to cover subsets of $D$ on which our eigenvalue condition (2.6) is violated.] More precisely, let $1 \leqq \alpha<2$, and $M \geqq 2$ be specified constants. We define

$$
\begin{aligned}
\mathscr{C}_{n}^{\prime} & \equiv \mathscr{C}_{n}^{\prime}(\alpha, M) \\
& \equiv\left\{c: c \in \mathscr{C}_{n}, \operatorname{dist}\left(c, c^{\prime}\right) \geqq M 2^{\alpha n}, \text { for all } c^{\prime} \in \mathscr{C}_{n}, c^{\prime} \neq c\right\}, \\
V_{n}^{\prime} & \equiv\left|\mathscr{C}_{n}^{\prime}\right|, \quad V^{\prime} \equiv \sum_{n=1}^{n_{0}} V_{n}^{\prime} .
\end{aligned}
$$

Theorem 4.4. For $\alpha<2, M<\infty$ there are finite constants $K_{0}(\alpha, M)$ and $K^{\prime}(\alpha, M)$ such that, for arbitrary collections $\mathscr{C}_{n}, n=0,1,2, \ldots$, with the properties described above,

$$
V \leqq K_{0}(\alpha, M) V_{0}+K^{\prime}(\alpha, M) V^{\prime}
$$

Moreover, $K^{\prime}(1, M)=2$.

Remarks. For $v=2$ a variant of this result has been proven in Sect. 3 of [8]. The arguments given there combined with Proposition 4.1 prove Theorem 4.4 for $v=2$. The extension to arbitrary dimension $v$ is immediate. (See also [7] for a discussion of this and related results when $v=1$.)

Sketch of Proof of Theorem 4.4. Let $\gamma(n)$ be defined to be the integer part of $\frac{1}{\alpha}\left(n-1-\log _{2}[2 M+3 \sqrt{v}]\right)$. The basic observation required in the proof is the following inequality: For $n>1+\log _{2}[2 M+3 \sqrt{v}]$,

$$
V_{n} \leqq \frac{1}{2} V_{\gamma(n)}+V_{\gamma(n)^{\prime}}^{\prime} \quad\left(\text { provided } V_{\gamma(n)} \geqq 2\right) .
$$

To prove (4.7) we define $\mathscr{C}_{m}^{\prime \prime}=\mathscr{C}_{m} \sim \mathscr{C}_{m^{\prime}}^{\prime}$, for all $m$, and note that, given an arbitrary cube $c \in \mathscr{C}_{\gamma(n)}^{\prime \prime}$, one can find a cube $c^{\prime} \in \mathscr{C}_{\gamma(n)}^{\prime \prime}, c^{\prime} \neq c$ such that

$$
\operatorname{dist}\left(c, c^{\prime}\right)<M 2^{\alpha \gamma(n)} \text {. }
$$

By the definition of $\gamma(n)$ and Proposition 4.1, $c$ and $c^{\prime}$ can be covered by a single cube in $\mathscr{C}_{n}$. More generally, if $c_{1}, \ldots, c_{l}$ are $l$ cubes belonging to $\mathscr{C}_{\gamma(n)}^{\prime \prime}$ such that for each $j \in\{1, \ldots, l\}$ there is a $\sigma(j) \neq j$ with the property that

$$
\operatorname{dist}\left(c_{j}, c_{\sigma(j)}\right)<M 2^{\alpha \gamma(n)},
$$

then one shows quite easily, using Proposition 4.1 , that [l/2] cubes in $\mathscr{C}_{n}$ suffice to cover $\left\{c_{1}, \ldots, c_{l}\right\}$, where $[l / 2]=$ integer part of $l / 2$. However, it will require $\left|\mathscr{C}_{\gamma(n)}^{\prime}\right|$ different cubes in $\mathscr{C}_{n}$ to cover all cubes in $\mathscr{C}_{\gamma(n)}^{\prime}$. Hence

$$
V_{n} \equiv\left|\mathscr{C}_{n}\right| \leqq \frac{1}{2}\left|\mathscr{C}_{\gamma(n)}^{\prime \prime}\right|+\left|\mathscr{C}_{\gamma(n)}^{\prime}\right| \leqq \frac{1}{2} V_{\gamma(n)}+V_{\gamma(n)}^{\prime},
$$

which is inequality (4.7). Given $n$, we can iterate (4.7) $N(n)$ times, where $N(n)$ is the largest integer for which $\gamma^{N(n)}(n) \geqq 0$. (Here $\gamma^{m}$ denotes the $m$-fold composition of the 
function $\gamma$ with itself.) Using the trivial fact that $V_{m} \leqq V_{0}$, for all $m>0$, we obtain by iterating (4.7)

$$
V_{n} \leqq\left(\frac{1}{2}\right)^{N(n)} V_{0}+\sum_{m=1}^{N(n)}\left(\frac{1}{2}\right)^{m-1} V_{\gamma^{m}(n)}
$$

By summing inequality (4.8) over all values of $n \leqq n_{0}$ and using simple properties of $\gamma$ and $N(n)$ which are discussed in detail in Sect. 3 of [8], Theorem 4.4 follows. [In particular, since $N(n) \sim\left(\frac{\log _{2} n}{\log _{2} \alpha}\right)+$ const, $\sum_{n=1}^{\infty}\left(\frac{1}{2}\right)^{N(n)} \leqq$ const $\left(\sum_{n=1}^{\infty}\left(\frac{1}{n}\right)^{1 / \log _{2} \alpha}\right)$ converges, provided $\alpha<2$.]

In certain applications to high- and low-temperature expansions in statistical mechanics it would be useful to have better constants in the bounds proven in Theorems 4.2 and 4.4 .

\section{Probability of Occurrence of Singular Sets}

\subsection{Statement of the Main Theorem}

Let $D$ be some finite subset of the lattice $\mathbb{Z}^{v}$. With $D$ we associate a subset $V_{D, j} \subset \Omega$, of potentials, $v$, as follows :

$$
V_{D, j}=\left\{v: D \text { is a component of } S_{j}^{g}(E ; v)\right\},
$$

where $S_{j}^{g}(E ; v)$ is the set of "gentle singular points of strength $j$," as constructed in Sect. 2.

The purpose of this section is to establish an upper bound on

$$
P_{D, j} \equiv \int_{V_{D, j}} d P(v) \equiv \operatorname{Prob}\left(V_{D, j}\right)
$$

Thus, let $v \in V_{D, j}$ and suppose, first, that

$$
\operatorname{diam} D \leqq d_{j-1} .
$$

Since $D$ is a component of $S_{j}^{g}$ and $S_{j} \subset S_{j-1}, D$ clearly satisfies Condition A(j-1), (a) and (b). Since the sets, $S_{i}^{g}, i=0,1,2, \ldots$, of singular points of strength $i$ are always chosen to be maximal, $D$ would be a component of $S_{j-1}^{g}$ if Condition A(j-1), (c) were satisfied. Thus, since $D \notin S_{j-1}^{g}$, it follows that

$$
\operatorname{dist}\left(\operatorname{spec} H_{\bar{D}}(v), E\right)<e^{-\sqrt{d_{j-1}}}
$$

for all $v \in V_{D, j}$, with $D$ satisfying (5.3). Here $\bar{D}=\left\{x \in \mathbb{Z}^{v}: \operatorname{dist}(x, D) \leqq 4 d_{j-1}\right\}$; see (2.7).

Second, if

$$
\operatorname{diam} D>d_{j},
$$

then $V_{D, j}$ is clearly empty, and hence

$$
P_{D, j}=0 \text {. }
$$


Let $\chi_{D, j}^{<}$be the characteristic function of the set of potentials

$$
\left\{v: \operatorname{dist}\left(\operatorname{spec} H_{D}(v), E\right)<e^{-\sqrt{1,}}, \operatorname{diam} D \leqq d_{i-1}\right\},
$$

and let $\chi_{D}$ be the characteristic function of the set, $V_{D}$, of potentials defined by

$$
\left\{v: \exists i \text { such that } D \text { is a component of } S_{i}^{g}(E, v)\right\} \text {. }
$$

Clearly $V_{D, j} \subset V_{D}$. Our discussion can now be summarized by the following inequality:

$$
P_{D, j} \leqq\left\{\begin{array}{lll}
\int \chi_{D, j}^{<}(v) \chi_{D}(v) d P(v), & \text { if } & \operatorname{diam} D \leqq d_{j-1} \\
P_{D} \equiv \int \chi_{D}(v) d P(v), & \text { if } & \operatorname{diam} D>d_{j-1} \\
0, & \text { if } & \operatorname{diam} D>d_{j}
\end{array}\right.
$$

If $\operatorname{diam} D \leqq d_{j-1}, P_{D, j}$ can be further estimated by

$$
P_{D, j} \leqq\left(P_{D, j}^{<}\right)^{1 / 2} P_{D}^{1 / 2}
$$

where

$$
P_{D, j}^{<} \equiv \int \chi_{D, j}^{<}(v) d P(v) .
$$

Inequality (5.9) follows from the first inequality in (5.8), by the Schwarz inequality. We have thus reduced the problem of estimating $P_{D, j}$ to estimating $P_{D}$ and $P_{D, j}^{<}$. By Lemma 2.4, if $|E-2 v|$ or $\delta$ is sufficiently large then

$$
P_{D, j}^{<} \leqq 2 \bar{\varrho}(E, \delta)\left(9 d_{j-1}\right)^{v} \exp \left(-\sqrt{d_{j-1}} / 2\right),
$$

where $\varrho(E, \delta) \rightarrow 0$ as $|E| \rightarrow \infty$ or $\delta \rightarrow \infty$. Thus it remains to estimate $P_{D}$.

Theorem 5.1. If $|E-2 v|$ or $\delta$ is sufficiently large

$$
P_{D} \leqq \exp \left[-k_{0}(E, \delta) V_{0}(D)-k^{\prime}(E, \delta) V^{\prime}(D)\right],
$$

where $V_{0}(D)$ and $V^{\prime}(D)$ are as defined in Sect. 4 , and $k_{0}, k^{\prime}$ are constants which tend to $+\infty$, as $|E| \rightarrow \infty$ or $\delta \rightarrow \infty$.

In Sect. 6 we shall combine the inequality in Theorem 5.1 with the entropy estimates of Sect. 4 to complete our probability estimates for the decay of the Green's function, $G(E+i \varepsilon, v ; x, y)$, announced in Theorems 1.2 and $1.2_{\Lambda}$ of the introduction.

\subsection{Proof of Theorem 5.1}

Let us first recall that $\mathscr{C}_{n}^{\prime}(D) \subset \mathscr{C}_{n}(D)$ is the set of isolated $n$-cubes $c$ with the property that $c \in \mathscr{C}_{n}^{\prime}(D)$ iff

$$
\operatorname{dist}(c, \tilde{c}) \geqq 2 \cdot 2^{(5 / 3) n},
$$

for all $\tilde{c} \in \mathscr{C}_{n}(D)$ with $\tilde{c} \neq c$. (We have set $M=2, \alpha=5 / 3$; see Sect. 4 for definitions.) The aim in Theorem 5.1 is to associate a small factor $e^{-k}$ with each isolated cube $c$ in $\mathscr{C}_{n}^{\prime}, n \geqq 1$. Roughly speaking, isolated subsets of $D$ are extremely unlikely, because on all such subsets the eigenvalue condition (2.6) must be violated. Lemma 2.4 can be used to show that this occurs with low probability, since $\kappa \simeq e^{-\sqrt{d_{n}}}$. 
(Note that if the eigenvalue condition were satisfied then $c \cap D$ would itself be a component belonging to $S_{m}^{g}, m<j$, because of our maximality condition.)

In order to make the above ideas more precise we shall first bound the characteristic function of $V_{D}$ by a product of characteristic functions. To define these characteristic functions let $j(n)$ be the smallest integer such that

$$
d_{j(n)} \equiv \exp \left[K_{0}\left(\frac{5}{4}\right)^{j(n)}\right] \geqq 2^{n} .
$$

If $c \in \mathscr{C}_{n}^{\prime}(D)$ we set

$$
\overline{c \cap D}=\left\{x: \operatorname{dist}(x, c \cap D) \leqq 4 d_{j(n)}\right\},
$$

and define $\chi_{n, c}(v)$ to be the characteristic function of

$$
\left\{v \mid \operatorname{dist}\left(\operatorname{spec} H_{\overline{c \cap D}}(v), E\right) \leqq \exp -\sqrt{d_{j(n)}}\right\} .
$$

When $n=0$ we set $\mathscr{C}_{j}^{\prime}(D)=D$ and identify $\mathcal{C}^{\prime} \in \mathscr{C}^{\prime}$ with a site in $D$. We define $\varkappa_{0 . c}$ to be the characteristic function of the set

$$
\{v:|v(c)-(E-2 v)| \leqq N(E, \delta)\},
$$

where $N(E, \delta)$ is defined after (2.2). Let

$$
I\left(K_{0}\right) \equiv\{0\} \cup\left\{n \in \mathbb{Z}: n \geqq K_{0} / \ln 2\right\}, \text { with } K_{0} \text { as in (2.4) . }
$$

Note that $j(n) \geqq 1$ for all $0 \neq n \in I\left(K_{0}\right)$. We adopt the convention that an empty product is defined to be 1 and that $\chi_{n, c}(l)=1$ for $n \notin I\left(K_{0}\right)$.

In Sect. 5.3 we shall prove the following lemma.

Lemma 5.2. For $\chi_{n, c}$ defined as above

$$
\chi_{V_{D}}(v) \leqq \prod_{n=0}^{\infty} \prod_{c \in \mathscr{C}_{n}^{\prime}(D)} \chi_{n, c}(v) .
$$

Assuming this lemma we shall prove Theorem 5.1 by using Hölder's inequality together with the statistical independence of $\chi_{n, c}$, for disjoint $c$ 's. In fact, if $c_{1}$ and $c_{2} \in \mathscr{C}_{n}^{\prime}(D)$ then

$$
\overline{c_{1} \cap D} \cap \overline{c_{2} \cap D}=\emptyset, \quad \text { if } c_{1} \neq c_{2},
$$

because

$$
\begin{aligned}
\operatorname{dist}\left(\overline{c_{1} \cap D}, \overline{c_{2} \cap D}\right) & \geqq 2 \cdot 2^{(5 / 3) n}-9 d_{j(n)} \\
& \geqq 2 \cdot 2^{(5 / 3) n}-9 \cdot 2^{(5 / 4) n} \\
& \geqq 0, \quad \text { if } K_{0} \text { is large enough. }
\end{aligned}
$$

This implies the independence of $\chi_{n, c}, c \in \mathscr{C}_{n}^{\prime}(D)$. Hence

$$
\int \prod_{c \in \mathscr{C}_{n}^{\prime}(D)} \chi_{n, c}(v) d P(v)=\prod_{c \in \mathscr{C}_{n}^{\prime}(D)} \int \chi_{n, c}(v) d P(v)
$$

Next we combine Lemma 5.2 and (5.19) with the Hölder inequality

$$
\left|\int f g d P\right| \leqq\left(\int|f|^{1 / r} d P\right)^{r}\left(\int|g|^{1 /(1-r)} d P\right)^{1-r}
$$


$0<r<1$, and use the obvious fact that $\chi^{q}=\chi, q>0$. This yields

$$
\begin{aligned}
\int \chi_{V_{D}}(v) d P(v) & \leqq \int\left\{\prod_{n=0}^{\infty} \prod_{c \in \mathscr{C}_{n}^{\prime}(D)}^{\infty} \chi_{n, c}(v)\right\} d P(v) \\
& \leqq\left[\prod_{c \in \mathscr{C}_{0}^{\prime}(D)} \int \chi_{0, c} d P\right]^{1-r} \cdot\left[\int\left\{\prod_{n=1}^{\infty} \prod_{c \in \mathscr{C}_{n}^{\prime}(D)} \chi_{n, c}\right\} d P\right]^{r} \\
& \leqq \ldots \\
& \leqq \prod_{n=0}^{\infty} \prod_{c \in \mathscr{C}_{n}^{\prime}(D)}\left[\int \chi_{n, c} d P\right]^{r^{n(1-r)}}
\end{aligned}
$$

by successive applications of (5.19) and (5.20).

Proof of Theorem 5.1. By the definition of $\chi_{n, c},(5.15)$, and the fact that $d_{j(n)} \geqq 2^{n}$, we see that Lemma 2.4, with $\kappa=\exp \left(-\sqrt{2^{n}}\right)$, implies

$$
\left[\int \chi_{n, c} d P\right]^{r^{n}(1-r)} \leqq \varrho(E, \delta)^{r^{n}(1-r)}|\overline{c \cap D}|^{r^{n}(1-r)} 2 \exp \left[(r-1)(r \sqrt{2})^{n} / 2\right] .
$$

We now choose $r=0.8>1 / \sqrt{2}$. Note that the right side of (5.22) is small when $n$ is large. When $n$ is small we use the fact that $\varrho(E, \delta)$ is small for $|E|$ or $\delta$ large. Therefore each factor in the product on the right side of (5.21) yields a small factor, for each cube in $\mathscr{C}_{n}{ }_{n}$, and Theorem 5.1 follows.

\subsection{Proof of Lemma 5.2}

Let $j(n)$ be given by (5.13), and $\overline{c \cap D}$ by $(5.14)$. For each $c \in \mathscr{C}_{n}^{\prime}(D)$ we propose to show that

$$
\operatorname{dist}\left(\operatorname{spec} H_{\overline{c \cap D}}(v), E\right) \leqq \exp \left(-\sqrt{d_{j(n)}}\right),
$$

for all $v \in V_{D}$, with $V_{D}$ as in (5.7). From this Lemma 5.2 clearly follows. Since $c \in \mathscr{C}_{n}^{\prime}(D)$, we have

$$
\begin{aligned}
\operatorname{diam}(D) & \geqq \operatorname{dist}\left(c, \mathscr{C}_{n}(D) \sim c\right) \geqq 2 \cdot 2^{(5 / 3) n} \\
& \geqq 2 \cdot 2^{(5 / 4)^{2} n} \geqq 2 \cdot d_{j(n)}^{5 / 4}
\end{aligned}
$$

and we have used (5.13).

Let $i(n)$ be the largest integer such that

$$
d_{i(n)} \equiv \exp \left[K_{0}(5 / 4)^{i(n)}\right] \leqq 2^{(5 / 3) n} .
$$

Then, by (5.24) and (2.4), we see that $D$ can be a component of $S_{k}^{g}(E ; v)$ only for some value of $k$ satisfying

$$
k>i(n) .
$$

If $j \leqq k$, and $C_{j}^{\alpha}$ is a component of $S_{j}^{g}(E ; v)$ different from $D$ then, by (2.5),

$$
\operatorname{dist}\left(c \cap D, C_{j}^{\alpha}\right) \geqq \operatorname{dist}\left(D, C_{j}^{\alpha}\right) \geqq 2 d_{j}^{(5 / 4)},
$$

and if $j>k$

$$
\begin{aligned}
& \operatorname{dist}\left(c \cap D, C_{j}^{\alpha}\right) \geqq \operatorname{dist}\left(D, S_{k} \sim D\right) \\
& \quad \geqq 2 d_{k+1}>2 d_{i(n)+1}>2 \cdot 2^{(5 / 3) n} \geqq 2 d_{j(n)}^{5 / 4} .
\end{aligned}
$$


Inequalities (5.24), (5.26), and (5.27) imply that $c \cap D$ satisfies Condition $A(j(n)),(a)$ and (b). Now recall that the sets $S_{j}^{g}(E ; v)$ are required to be maximal. Thus if $c \cap D$ satisfied Condition $\mathrm{A}(\mathrm{j}(\mathrm{n}))$, (c) it would be a component of $S_{j(n)}^{g}(E ; v)$. Since $j(n)<i(n)<k,(5.23)$ now follows.

It should be pointed out that, for each $c \in \mathscr{C}_{n}^{\prime}(D)$, the integer $j(n)$ and the validity of (5.23) do not depend on the potential $v$, as long as $v \in V_{D}$.

\section{Proofs of Theorems 2.2 and 2.3, Conclusions}

In this section we use the results of Sects. 4 and 5 and Theorem 2.1, proven in Sect. 3, to complete the proofs of Theorems 2.2 and 2.3. We recall that it has been shown in Sect. 2 that our main technical result, Theorem $1.2_{\Lambda}$ of Sect. 1 , follows from Theorem 2.3 and Lemma 2.4. A proof of Lemma 2.4 and related matters are contained in Appendices A and C.

The main points which remain to be discussed are the following:

(1) Let $\sigma \in(0,1)$ and $l \gg 1$ be given. Let $k$ be the largest integer for which

$$
\frac{1}{5} d_{k} \leqq l^{\sigma} .
$$

We must estimate the probability, $P(l, \sigma)$, that there exists a $(k-1)$-admissible region $A$ containing 0 such that

$$
l / 2 \leqq \min _{b \in \partial A} \operatorname{dist}(0, b) \leqq \max _{b \in \partial A} \operatorname{dist}(0, b) \leqq l,
$$

and with the property that

$$
A \cap S_{k}(E ; v)=\emptyset .
$$

For such regions it follows from Theorem 2.1 that

$$
\left|G_{A}(E+i \varepsilon, v ; x, y)\right| \leqq e^{-m|x-y|},
$$

with

$$
m=m(E, \delta) \geqq \frac{1}{2} \ln (N(E, \delta))
$$

provided

$$
|x-y| \geqq l^{\sigma} \geqq \frac{1}{5} d_{k} .
$$

Theorem 2.3 follows if we can show that given any $p<\infty$, there is a finite constant $c_{p, \sigma}$ such that, for $|E|+\sqrt{\delta} \geqq c_{p, \sigma}$,

$$
P(l, \sigma) \geqq 1-l^{p} \text {. }
$$

Our proof of this estimate proceeds as follows:

First we notice that, with probability 1 , there exists a $(k-1)$-admissible region $A$ satisfying (6.2). This is shown in Appendix D.

Thus it is enough to estimate the conditional probability, $P_{k}$, that $A \cap S_{k}(E ; v)=\emptyset$, given that $A$ is a $(k-1)$-admissible set satisfying (6.2). But $P_{k}$ can be bounded below by the probability $P_{h}(l)$ that any set $A$ which satisfies (6.2) does not intersect $S_{h}(E: \imath)$. Clearly

$$
P_{h}(l) \geqq 1-\text { const } l^{v} \max _{|.| \leqq i}\left(1-P_{\lambda, h}\right)
$$


where $P_{x, k}$ is the probability that $x \notin S_{k}(E ; v)$. Since the distribution $d P(v)$ of potentials $v$ is translation-invariant,

$$
\min _{|.| \leqq i} P_{\lambda . h}=P_{0 . h} .
$$

Next, we bound $P_{0, k}$ from below by

$$
P_{0, k} \geqq 1-\sum_{j=k}^{\infty} p_{j}-p_{\infty},
$$

where $p_{j} \equiv \operatorname{Prob}\left(V_{0, j}\right)$ is the probability that $0 \in S_{j}^{g}(E ; v)$. Here

$$
V_{x, j} \equiv\left\{v: S_{j}^{g}(E ; v) \ni x\right\},
$$

see (2.15), and $p_{\infty}$ is the probability that

is non-empty.

$$
S_{\infty} \equiv \bigcap_{k=0}^{\infty} S_{k}=S_{0} \sim\left(\bigcup_{k=0}^{\infty} S_{k}^{g}\right)
$$

Thus, we must show

(2) for arbitrary finite regions $\Lambda$

$$
\operatorname{Prob}\left\{v: S_{\infty} \equiv S_{\infty}\left(E ;\left.v\right|_{A}\right)=\emptyset\right\}=1,
$$

i.e. $p_{\infty}=0$; and

(3) given an arbitrary $q<\infty$, there is a finite constant $c_{q}$ such that

if $|E|+V \bar{\delta} \geqq c_{q}$.

$$
p_{j} \leqq d_{j}^{-q}
$$

Remarks. 1) We note that (3) clearly yields Theorem 2.2 .

2) From (6.9), (2) and (3) it follows that

$$
P_{0, k} \geqq 1-\text { const } d_{k}^{-q}, \quad q>0,
$$

(because $p_{j} \leqq d_{j}^{-q}=\exp \left[-q K_{0}(5 / 4)^{j}\right]$ ). Now, recall that $k$ is the largest integer with the property that

$$
\frac{1}{5} d_{k} \leqq l^{\sigma}, \quad \text { i.e., } \quad d_{k+1}=d_{k}^{5 / 4} \geqq 5 l^{\sigma} .
$$

Thus

$$
P_{0, k} \geqq 1-\text { const } l^{-\frac{4}{5} \sigma q} .
$$

By the definition of $P(l, \sigma),(6.7),(6.8)$, and (6.11), it now follows that

$$
\begin{aligned}
P(l, \sigma) & \geqq 1-\text { const } l^{\nu-\frac{4}{5} \sigma q} \\
& \geqq 1-l^{-p},
\end{aligned}
$$

for any finite $p$, If $|E|+\sqrt{\delta} \geqq c_{q}$, with $q>\frac{5}{4 \sigma}(p+v)$. This proves our basic lower bound on $P(l, \sigma)$, see (6.6). Thus the proof of Theorem 2.3 is complete.

We now turn to our proofs of (2) and (3); see (6.10). Point (2) is settled easily: Since $\Lambda$ is assumed to be a finite region, Conditions A(i), (a) and (b) of Sect. 2 will 
be satisfied for $i$ large enough. Thus for $S_{\infty}$ to be non-empty it is necessary that $E$ is an eigenvalue of $H_{\Lambda}=-\Delta_{\Lambda}+v \chi_{\Lambda}$. Since $\operatorname{spec} H_{\Lambda}$ is discrete, this event has probability 0 .

To settle (3) we use the results in Sects. 4 and 5: To begin, note that

$$
p_{j} \equiv \operatorname{Prob}\left(V_{0, j}\right) \leqq \sum_{D \ni 0} P_{D, j}
$$

where $P_{D, j}$ is the probability that the set $D \subset \mathbb{Z}^{v}$ is actually a component of $S_{j}^{g}(E ; v)$. Thus we need an estimate on $P_{D, j}$. Such an estimate has been established in Sect. 5. Let $V(D)$ and $V^{\prime}(D)$ be defined as in Sect. 4 ; see (4.2) and (4.6). Then, by (6.12) and inequality (5.8),

$$
\begin{aligned}
p_{j} & \leqq \sum_{V=1}^{\infty} \sum_{\begin{array}{c}
D \ni 0 \\
V(D)=V
\end{array}} P_{D, j} \\
& \leqq \sum_{V=1}^{\infty}\left\{\sum_{\begin{array}{c}
D \ni 0 \\
V(D)=V \\
\operatorname{diam} D \leqq d_{j-1}
\end{array}}\left(P_{D, j}^{<}\right)^{1 / 2} P_{D}^{1 / 2}+\sum_{\begin{array}{r}
D \ni 0 \\
V(D)=V \\
\operatorname{diam} D \geqq d_{J-1}
\end{array}} P_{D}\right\}
\end{aligned}
$$

where $P_{D, j}^{<}$has been defined in (5.10) and $P_{D}$ in (5.8). The second inequality in (6.13) follows from (5.8). By (5.11),

and by Theorem 5.1,

$$
\left.\begin{array}{c}
P_{D, j}^{<} \leqq 2 \bar{\varrho}(E, \delta)\left(9 d_{j-1}\right)^{v} e^{-\sqrt{d_{j-1}} / 2} \equiv \Phi_{j}(E, \delta), \\
P_{D} \leqq \exp \left[-k_{0}(E, \delta) V_{0}(D)-k^{\prime}(E, \delta) V^{\prime}(D)\right],
\end{array}\right\}
$$

if $|E|+\sqrt{\bar{\delta}}$ is large enough. [Here $V_{0}(D) \equiv|D|$ is the number of sites in $D$.]

Next, we use Theorem 4.4 , with $\alpha=\frac{5}{3}$ and $M=2$, to conclude that

$$
V(D) \leqq K_{0} V_{0}(D)+K^{\prime} V^{\prime}(D)
$$

so that, by (6.14),

$$
P_{D} \leqq \exp [-K(E, \delta) V(D)],
$$

for some constant $K(E, \delta)$ which tends to $+\infty$, as $(|E|+\delta) \rightarrow \infty$. It is an immediate consequence of the definition of $V$ (see Sect. 4) that

$$
V(D) \geqq \text { const } \ln \operatorname{diam} D \text {. }
$$

Thus, by (6.13)-(6.16),

$$
p_{j} \leqq \sum_{V} \Phi_{j}(E, \delta)^{1 / 2} e^{-K(E, \delta) V / 2} N(V)+\sum_{V \geqq \text { constlnd } d_{j}} e^{-K(E, \delta) V} N(V)
$$

where

$$
N(V) \equiv \#\{D: D \ni 0, V(D)=V\}
$$

By Theorem 4.2,

$$
N(V) \leqq e^{K_{v} V}
$$


Thus

$$
p_{j} \leqq \Phi_{j}(E, \delta)^{1 / 2} \sum_{V} e^{\left(K_{v}-\frac{1}{2} K(E, \delta)\right) V}+\sum_{V \geqq \text { const Ind } d_{j}} e^{\left(K_{v}-K(E, \delta)\right) V} .
$$

By (6.14) and (6.17),

$$
p_{j} \leqq\left(1 / d_{j}\right)^{q(E, \delta)},
$$

with $q(E, \delta) \rightarrow \infty$, as $|E|+\delta \rightarrow \infty$. This proves (3).

The proofs of the results announced in Sects. 1 (Theorems 1.1 and 1.2) and 2 (Theorem 2.3) are now complete.

To conclude, we wish to mention some interesting open problems.

1) It appears to be rather straightforward to extend our analysis to systems with off-diagonal disorder, or to the problem of wave propagation in a random environment on $\mathbb{Z}^{v}$, provided the disorder is large (or the energy is constrained to a suitable region).

2) It would be interesting to improve our methods in such a way that not only absence of diffusion (for large disorder or at low energies), but localization, in the strong sense of the word, is obtained, i.e.

$$
\operatorname{spec} H \cap I=\sigma_{p p}(H) \cap I \neq \emptyset
$$

if $I$ is chosen to be a union of intervals located at sufficiently large values of $|E|$ (and if the disorder is large, $I$ is the whole real line). A proof of this requires mastering more serious small divisor problems. While we have envisaged a tentative strategy to get at such results we do not know of any complete analysis.

3) As the reader has noticed, estimates on the density of states are an important ingredient of our analysis. It would be interesting to improve the present estimates on the density of states (see $[9,10])$ and, for example, establish bounds on and smoothness properties of $\varrho(E)$, for $E$ in the region where extended states are expected.

4) It would be interesting to prove general upper bounds on the diffusion constant $D(E)$, for arbitrary $E$. This will probably require new ideas.

5) It may be possible to apply our methods to almost periodic Schrödinger operators on $\mathbb{Z}^{v}$. In particular one might consider a limit periodic potential with random coefficients. A primary problem in an analysis of almost periodic operators will be control over the density of states.

\section{Appendix A: The Path Expansion for $G$}

In this appendix we expand the Green's function in the off diagonal matrix elements of $-\Delta$ to obtain a formal series for $G_{A}(E)$. The terms of the series are indexed in a natural way by paths $\omega$ which represent a sequence of nearest neighbor pairs of lattice sites in $\mathbb{Z}^{v}$. If $D$ is the diagonal operator

$$
D_{i j}=\left(2 v+v_{j}-E-i \varepsilon\right)^{-1} \delta_{i j},
$$

and $J$ denotes the off diagonal elements of $\Delta_{\Lambda}$, i.e.

$$
J_{i j}=1 \quad \text { if } \quad|i-j|=1, \quad i, j \in \Lambda,
$$


and 0 otherwise. Then formally, see $[1,14]$

$$
\begin{aligned}
G_{\Lambda}(E+i \varepsilon, v ; x, y) & =\left[D^{-1}-J\right]^{-1}(x, y) \\
& =\sum_{n=0}^{\infty}\left[D(J D)^{n}\right](x, y) \\
& =\sum_{\omega: x \rightarrow y} \prod_{j \in \Lambda} D_{j j}^{n_{j}(\omega)},
\end{aligned}
$$

where $\omega$ ranges over nearest neighbor paths starting at $x$ and ending at $y$ which are constrained to lie in $\Lambda$. Also, $n_{j}(\omega)$ denotes the number of times $\omega$ visits the lattice site $j \in \Lambda$. Note that if $S_{0} \cap A=\emptyset$ then $\left|D_{j}\right| \leqq \frac{1}{N}, N \equiv N(E, \delta)$ [see (2.2) for the definition of $N(E, \delta)]$ and

$$
\begin{aligned}
\left|G_{\Lambda}(E ; x, y)\right| & \leqq \sum_{\omega: x \rightarrow r} \prod_{j \in \Lambda}\left(\frac{1}{2 v+\varepsilon}\right)^{n,(\omega)}\left(\frac{2 v+\varepsilon}{N}\right)^{n ;(\omega)} \\
& \leqq\left|\frac{2 v+\varepsilon}{N}\right|^{|.-1|} \sum_{\omega: \Lambda \rightarrow r} \prod_{j \in \Lambda}\left(\frac{1}{2 v+\varepsilon}\right)^{n,(\omega)} \\
& =\left|\frac{2 v+\varepsilon}{N}\right|^{|x-y|}(-\Delta+\varepsilon)^{-1}(x, y)
\end{aligned}
$$

with $\varepsilon$ chosen such that $\left|\frac{2 v+\varepsilon}{N}\right| \leqq 1$. [This is possible if an appropriate definition of $N(E, \delta)$ is made.]

In general, it is clear that the factors $D_{j j}$ may become arbitrarily large, and (A.1) will not be absolutely convergent. This is the problem of small divisors. Our strategy is to selectively expand the off diagonal matrix elements of $\Delta$, depending upon $v$. In this way we obtain a convergent series.

Representation (A.1) is also useful for estimates on the density of states, see (1.11),

$$
\begin{aligned}
\varrho(E) & =\lim _{\varepsilon \rightarrow 0} \mathscr{I} m \int d P(v) G(E+i \varepsilon, v ; 0,0) \\
& =\lim _{\varepsilon \rightarrow 0} \mathscr{I}_{m} \sum_{\omega: 0 \rightarrow 0} \prod_{j} \int D_{j j}^{n_{j}(\omega)} d \lambda\left(v_{j}\right) .
\end{aligned}
$$

If $d \lambda\left(v_{j}\right)$ is, for example, the Gaussian given by (1.14), then by deforming the contour of integration so as to avoid the zero of $D_{j}^{-1}$, it is easy to show that if $|E-2 v|$ is large or $\gamma$ is large then

$$
\left|\int D_{j j}^{n_{j}(\omega)} d \lambda\left(v_{j}\right)\right| \leqq a^{n_{j}(\omega)},
$$

where $a=a(E, \gamma) \rightarrow 0$ as either $|E-2 v|$ or $\gamma$ become large. Thus if $|a|<(2 v)^{-1}$ the integrated series converges and $\varrho(E)$ is bounded and analytic in $E$. Similarly one can prove that the average Green's function decays in $|x-y|$ for large $|E|$. In order to make this argument rigorous it is important to first take $\varepsilon$ large so that the series (A.1) is manifestly convergent. We then let $\varepsilon \rightarrow 0$ after integration. Moreover one can obtain with some extra effort good bounds on $\varrho(E)$ as $|E-2 v| \rightarrow \infty$ (see $[9,14]$ for details). 
We remark that the above analysis breaks down if one tries to estimate the average of the absolute value of the Green's function because deformation of our contour is no longer possible.

\section{Appendix B : Proof of (1.28)}

Let $U(a)$ be the multiplication operator on $l_{2}\left(\mathbb{Z}^{\nu}\right)$ given by $e^{i a \cdot j}$. By direct calculation we see that

$$
U(-a) H_{\Lambda}(v) U(a)=H_{\Lambda}(v)+Q_{\Lambda}(a),
$$

where $Q_{\Lambda}(a)$ is a bounded operator independent of $l$ which has an analytic continuation in a satisfying

$$
\left\|Q_{\Lambda}(a)\right\| \leqq C|a|
$$

for $a \in \mathbb{C}^{v}$ and $|a| \leqq 1$. If we choose $|a| \leqq \frac{\varepsilon}{2 C}$ then

$$
U(--a) G_{\Lambda}(E+i \varepsilon) U(a)=\left[H_{\Lambda}-E+i \varepsilon Q_{\Lambda}(a)\right]^{-1}
$$

is a bounded operator on $l_{2}\left(\mathbb{Z}^{v}\right)$ with norm less than $2 \varepsilon^{-1}$. Now observe that the $x, y$ matrix element of (B.2) which equals

$$
e^{i a \cdot(x-y)} G_{\Lambda}(E+i \varepsilon ; x, y)
$$

must be bounded in absolute value by $2 \varepsilon^{-1}$. This completes our proof of (1.28).

Remarks. A similar result holds if there is a gap in the spectrum of $H$, with $\varepsilon$ playing the role of the width of the gap.

In the above we have relied on the so-called Combes-Thomas argument (see [15] and references therein).

\section{Appendix C: Bounds on Density of States}

Proof of Lemma 2.4. Let $\chi_{\Lambda}(\kappa, E)$ be the characteristic function of the set

$$
\left\{v \mid \operatorname{dist}\left(\operatorname{spec} H_{\Lambda}(v), E\right) \leqq \kappa\right\},
$$

and let $N_{\Lambda}(E, v)$ be as in (1.10). Following Wegner [10],

$$
\begin{aligned}
\int \chi_{\Lambda}(\kappa, E) d P & \leqq \int\left[N_{\Lambda}(E+\kappa, v)-N_{\Lambda}(E-\kappa, v)\right] d P \\
& =\int_{\left|E^{\prime}-E\right| \leqq \kappa} \frac{d}{d E^{\prime}} N_{\Lambda}\left(E^{\prime}, v\right) d E^{\prime} d P \\
& =\sum_{j}-\int_{\left|E^{\prime}-E\right| \leqq \kappa} \frac{d}{d v_{j}} N_{\Lambda}\left(E^{\prime}, v\right) d E^{\prime} d P \\
& \leqq \sum_{j} \sup _{v_{j}}\left(\frac{d \lambda\left(v_{j}\right)}{d v_{j}}\right) \int \delta_{j} N_{\Lambda}\left(E^{\prime}, v\right) d E^{\prime} \prod_{k \neq j} d \lambda\left(v_{k}\right),
\end{aligned}
$$

where

$$
\delta_{j} N_{\Lambda}\left(E^{\prime}, v\right) \equiv N_{\Lambda}\left(E^{\prime}, v^{-}\right)-N_{\Lambda}\left(E^{\prime}, l^{+}\right)
$$


and $v_{k}^{ \pm}=v_{k}$ for $k \neq j$, and $v_{j}^{+}>v_{j}^{-}$denote the endpoints of the support of $d \lambda$ (possibly $\pm \infty$ ). Note that the integrands above are all nonnegative and that we have used the relation $N(E, v)=N(0, v-E)$. Since $v_{j}$ is a rank one perturbation of $H_{A}(v)$ it follows from the minimax principle that the eigenvalues of $H_{A}(v)$ are interlaced $\lambda_{k}^{-} \leqq \lambda_{k}^{+} \leqq \lambda_{k+1}^{-}$, hence $0 \leqq \delta_{j} N \leqq 1$, a.e. in $v$ (since the $\lambda_{k}$ are distinct with probability 1). Thus

$$
\int \chi_{\Lambda}(\kappa, E) \leqq 2 \kappa \delta^{-1}|\Lambda|
$$

This establishes Lemma 2.4 if $\delta$ is large.

If $|E|$ is large, $N_{A}(E, v) \geqq 1$ only if for at least one $j, \mid v,-E+2 v \leqq 2 v$ which occurs with probability less than

$$
|\Lambda| \int_{|V-E+2 v| \leqq 2 v} d \lambda(v)
$$

When $d \lambda(v)$ is Gaussian, then the integral is small for large E. Lemma 2.4 follows by taking the geometric mean of (C.4) and (C.5).

Remark. We have used only the fact that $\frac{d \lambda(v)}{d v}$ is bounded and that for large $|E|$ (C.5) is small. If we assume that $\lambda\left(v_{j}\right)$ is Gaussian then the factor $\sqrt{2 \kappa}$ which appears in Lemma 2.4 may be replaced by $2 \kappa$. This follows from the method of Appendix A (see also [9]).

\section{Appendix D: Admissible Sets}

Lemma. Let $D_{2} \supset D_{1}$ be rectangular regions such that

$$
\operatorname{dist}\left(D_{1}, \sim D_{2}\right) \geqq 10 d_{k} .
$$

If $d_{0}$ is sufficiently large, then there is a k-admissible set $A$ such that $D_{2} \supset A \supset D_{1}$.

Remarks. The admissible sets needed in Sects. 3 and 6 are given by the above lemma after a suitable choice of $D_{1}$ and $D_{2}$.

The proof of this lemma is nearly the same as the argument which appears in Appendix E of [8].

Proof. Let

$$
\tilde{C}_{j}^{\alpha}=\left\{x \in \mathbb{Z}^{v}: \operatorname{dist}\left(x, \overline{C_{j}^{\alpha}}\right) \leqq 2\right\}
$$

and let

$$
U=\bigcup_{\alpha, j \leqq k} \tilde{C}_{j}^{\alpha} .
$$

Now consider the components of $U$ (defined in the usual nearest neighbor sense) which meet $\partial D_{1}-$ call them $U^{\beta}$. We claim that

$$
\operatorname{diam}\left(U^{\beta}\right) \leqq 10 d_{r},
$$

where $r$ is the largest integer such that $U^{\beta} \supset \tilde{C}_{r}^{\alpha}$ for some $\alpha$. Let $A$ be defined as the union of $D_{1}$ and $\bigcup_{\beta} U^{\beta}$. Clearly (D.3) implies that $A \subset D_{2}$.

We shall establish (D.3) by induction on $r$. Clearly (D.3) holds if $r \leqq 1$ using (2.4) and (2.5). Now let $U^{\beta}$ and $r>1$ be given and let $\tilde{C}_{r}^{\alpha_{r}}, i=1,2, \ldots, m$ denote those $\tilde{C}_{r}^{\alpha}$ 
contained in $U^{\beta}$. We define $Q^{\gamma}$ to be the components of

By induction,

$$
U^{\beta} \sim \bigcup_{i}^{m} \tilde{C}_{r}^{\alpha_{i}}
$$

$$
\operatorname{diam} Q^{\gamma} \leqq 10 d_{r-1}
$$

which is much less than

$$
\operatorname{dist}\left(\tilde{C}_{r}^{\alpha_{i}}, \tilde{C}_{r}^{\alpha_{J}}\right) \geqq 2 d_{r+1}-8 d_{r}-4
$$

for $d_{0}$ large. In the last inequality we have used (2.5), (2.7), and (D.2). Therefore each $Q^{\gamma}$ can meet at most one $\tilde{C}_{r}^{\alpha_{2}}$. Since $U^{\beta}$ is connected, it follows that $U^{\beta}$ contains exactly one $C_{r}^{\alpha_{1}}($ i.e. $m=1)$. Therefore if $d_{0}$ is sufficiently large,

$$
\begin{aligned}
\operatorname{diam} U^{\beta} & \leqq \operatorname{diam} \bar{C}_{r}^{\alpha_{1}}+20 d_{r-1} \\
& \leqq 9 d_{r}+4+20 d_{r-1} \\
& \leqq 10 d_{r} .
\end{aligned}
$$

Acknowledgement. We wish to thank Arthur Jaffe for his hospitality at Harvard University where much of this paper was written.

\section{References}

1. Anderson, P.: Absence of diffusion in certain random lattices. Phys. Rev. 109, 1492 (1958)

2. Goldsheid, I., Molchanov, S., Pastur, L.: Pure point spectrum of stochastic one-dimensional Schrödinger operators. Funct. Anal. App. 11, 1 (1977)

3. Kunz, H., Souillard, B.: Sur le spectre des opérateurs aux différences finies aléatoires. Commun. Math. Phys. 78, 201-246 (1980)

4. Carmona, R. : Exponential localization in one-dimensional disordered systems. Duke Math. J. 49, 191 (1982)

5. Delyon, F., Kunz, H., Souillard, B.: One dimensional wave equation in disordered media. Preprint 1982, Ecole Polytechnique

6. Kunz, H., Souillard, B.: To appear

7. Fröhlich, J., Spencer, T.: The phase transition in the one-dimensional Ising model with $1 / r^{2}$ interaction energy. Commun. Math. Phys. 84, 87 (1982)

8. Fröhlich, J., Spencer, T.: The Kosterlitz-Thouless transition in two-dimensional abelian spin systems and the Coulomb gas. Commun. Math. Phys. 81, 527 (1981); Kosterlitz-Thouless transition in two-dimensional plane rotator and Coulomb gas. Phys. Rev. Lett. 46, 1006 (1981)

9. Constantinescu, F., Fröhlich, J., Spencer, T.: To appear

10. Wegner, F.: Bounds on the density of states in disordered systems. Z. Phys. B 44, 9-15 (1981)

11. McKane, A., Stone, M. : Localization as an alternative to Goldstone's theorem. Ann. Phys. 131, 36 (1981)

12. Ruelle, D.: A remark on bound states in potential-scattering theory. Nuovo Cimento A 61, 655 (1969)

13. Simon, B.: Correlation inequalities and the decay of correlations in ferromagnets. Commun. Math. Phys. 77, 111 (1980)

14. Edwards, S., Thouless, D.: Regularity of the density of states in Anderson's localized electron model. J. Phys. C 4, 453 (1971)

15. Reed, M., Simon, B.: Methods of modern mathematical physics. IV. New York: Academic Press 1978

Communicated by A. Jaffe

Received November 15, 1982 